Accepted for publication in PASP

\title{
A contribution to the selection of emission-line galaxies using narrow-band filters in the optical airglow windows
}

\author{
S. Pascual and J. Gallego and J. Zamorano \\ Departamento de Astrofísica y Ciencias de la Atmósfera, Facultad de C.C. Físicas, \\ Universidad Complutense de Madrid, E-28040, Madrid, Spain \\ spr@astrax.fis.ucm.es
}

\begin{abstract}
Emission line galaxies are an invaluable tool for our understanding of the evolution of galaxies in the Universe. Imaging of deep fields with narrow-band filters allows not only the selection of these objects, but also to infer the line flux and the equivalent width of the emission line with some assumptions. The narrowband filter technique provides homogeneous samples of galaxies in small comoving volumes in the sky. We present an analysis of the selection of emission-line galaxies using narrow-band filters. Different methods of observation are considered: broad-band - narrow-band filters and two broad-band and one narrow-band filters. We study also the effect of several lines entering simultaneously inside the filters (this is the case of $\mathrm{H} \alpha$ ). In each case the equations to obtain the equivalent width and line flux from the photometry are obtained. Candidates to emissionline objects are selected by their color excess in a magnitude-color diagram. For different narrow-band filters, we compute the mean colors of stars and galaxies, showing that, apart from galaxies, some types of stars could be selected with certain filter sets. We show how to compute the standard deviation of the colors of the objects even in the usual case when there are not enough objects to determine the standard deviation from the data. We present also helpful equations to compute the narrow-band and the broad-band exposure times in order to obtain minimum dispersion in the ratio of fluxes of both bands with minimum total exposure time.
\end{abstract}

Subject headings: techniques: photometric — stars: emission-line, Be — galaxies: fundamental parameters 


\section{Introduction}

Emission line galaxies (hereafter ELGs) are an invaluable tool for our understanding of the evolution of galaxies in the Universe. Faint galaxies can be hard to confirm spectroscopically, while the ELGs are generally easy to identify. Furthermore, the emission lines are produced within regions related either with star formation or with the active galactic nuclei (AGN) phenomena.

Before the advent of CCD detectors, surveys for emission-line galaxies used photographic plates with objective prisms in Schmidt telescopes (e.g. Smith 1975; Smith et al. 1976; MacAlpine et al. 1977; Wasilewski 1983; Pesch \& Sanduleak 1983; Salzer et al. 1989; Zamorano et al. 1994, 1996).

Several low redshift surveys have made use of the objective-prism technique with CCD detectors to circumvent the limit on wavelength and quantum efficiency imposed by photographic plates (e.g., Sabbey et al. 2001; Gronwall et al. 2004; Bongiovanni et al. 2005). However the objective prism, as any system of slitless spectroscopy, blends the flux of the object with that of the dispersed sky, reducing the sensitivity of the survey for the objects with less contrast between the continuum and the line (that is, with small equivalent width). This effect, together with relatively small aperture of the telescopes used, limits these surveys to detect bright sources in a near volume of the space.

A more efficient approach to detect ELGs is to use narrow-band imaging (Djorgovski et al. 1985; Lowenthal et al. 1991; Macchetto et al. 1993; Thompson et al. 1995). This technique reduces significantly the contribution of the sky brightness, as it is admitted in a small range of wavelengths. The sky background, that is the most significant limitation in the detection of objects in deep broad band images, is greatly reduced. A small wavelength range increases also the contrast between the emission line and the continuum. Narrow-band imaging has

been used also to detect both galactic (Rauch 1999) as well as extragalactic (Okamura et al. 2002; Castro-Rodríguez et al. 2003; Arnaboldi et al. 2003) planetary nebulae.

In order to use the narrow pass-band filters with maximum efficiency, regions of the night sky spectrum with a minimum background are selected. In the optical wavelengths, the windows in the Meinel OH bands around $8200 \AA$ and $9200 \AA$ have been used to center narrow-band filters. Most of the interest has gone to the region around $8200 \AA$, where the CCD detectors still have a good quantum efficiency.

Similar to the narrow-band imaging is the use of a tunable filter (TF, Bland-Hawthorn \& Jones 1998). A tunable filter works like a Fabry-Perot interferometer modified to produce a wide region of interference and to cover a range of wavelengths. 
Compared with narrow-band imaging, a TF provides a pseudo-spectrum, when the spectral range is explored with small wavelength steps. As a disadvantage, the TF requires a dedicated instrument, whereas narrow-band imaging demands only the narrow-band filter and can be carried out in any imaging facility. Both narrow-band imaging and TFs need very long exposure times to achieve the necessary signal-noise ratio.

The search for emission-line galaxies using a given narrow-band filter is open to different lines at different redshifts. Lines whose redshifted wavelength fall inside the narrow-band filter range could be detected. When the emission-line is bright enough the galaxy will be selected. The more prominent lines that can be detected in the $8200 \AA$ and $9200 \AA$ atmospheric windows are $\mathrm{H} \alpha \lambda 6563$ (at redshift $z \simeq 0.24$ and $z \simeq 0.4$ ), [OIII] $\lambda \lambda 4959,5007$ ( $z \simeq 0.6$ and $z \simeq 0.8),[\mathrm{OII}] \lambda 3727(z \simeq 1.2$ and $z \simeq 1.5)$ and $\operatorname{Ly} \alpha \lambda 1216(z \simeq 5.7$ and $z \simeq 6.5)$.

The $\mathrm{H} \alpha$ line is an excellent tracer of the Star Formation Rate (SFR, Kennicutt 1998; Charlot \& Longhetti 2001), provided that the ionizing flux comes from young stars and not from non-thermal activity. Other commonly used SFR tracers are the far infrared (FIR) and the UV. The three tracers share sensitivity to the parameters of the Initial Mass Function. $\mathrm{H} \alpha \mathrm{SFR}$ is affected by obscuration but is not very sensitive to metallicity. The FIR is not affected by obscuration, but there is uncertainty in how the total $(8-1000 \mu \mathrm{m})$ luminosity is computed from monochromatic measurements (e.g. Spitzer's $24 \mu \mathrm{m}$ ) and the contribution of different non-SFR related components (cirrus, hot dust) to the FIR. Finally the UV is heavily affected by obscuration. As shown by Bell (2003), obscuration corrected $\mathrm{H} \alpha$ is consistent, to a factor of 2, with the summed SFRs estimated using UV and FIR $(8-1000 \mu \mathrm{m})$. Consequently, $\mathrm{H} \alpha$ observations of galaxy samples with UV and FIR data provides an invaluable tool to understand the evolution of the SFR and the role of obscuration in the determination of global SFR for galaxies. Some works have used narrow-band imaging to select $\mathrm{H} \alpha$ candidates

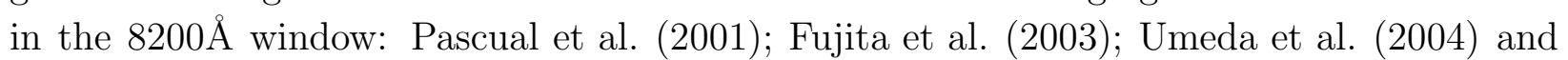
Ajiki et al. (2006b).

Given that small amounts of dust reddening can completely extinguish Ly $\alpha$-emission, $\mathrm{H} \alpha$ is the strongest emission line in many starburst galaxies. The [OIII] $\lambda \lambda 4959,5007$ doublet is in many cases the other strongest emission line. In fact, the $[\mathrm{OIII}] \lambda \lambda 4959,5007 / \mathrm{H} \alpha$ ratio is 0.6 in local star-forming galaxies (Mouhcine et al. 2005). This line is not used to trace the star formation but to trace the power of AGN (see, for example Kauffmann et al. 2003). Thus, [OIII] $\lambda \lambda 4959,5007$ emitters can be a tool to unveil AGN at different redshifts.

$[\mathrm{OII}] \lambda 3727$ is also intense when compared with $\mathrm{H} \alpha$, with a ratio [OII] $\lambda 3727 / \mathrm{H} \alpha=0.45$ (Kennicutt 1992). However, the ratio depends on luminosity and metallicity (Jansen et al. 2001) and varies from sample to sample. This line is used as a star-formation indicator at redshifts where $\mathrm{H} \alpha$ is outside the visible range (which happens at $z>0.4$ ). SFR based 
on $[\mathrm{OII}] \lambda 3727$ comes from the fact that there is a good correlation between [OII] $\lambda 3727$ emission and $\mathrm{H} \alpha$ emission. [OII] $] 3727$ is used as a proxy for the $\mathrm{H} \alpha$ emission. Different calibrations of SFR based on $\mathrm{H} \alpha$ are used (see, e.g. Gallagher et al. 1989; Kennicutt 1998; Rosa-González et al. 2002; Kewley et al. 2004) but, in general, the correlation between SFR and $[\mathrm{OII}] \lambda 3727$ is affected intensely by extinction and metallicity (Mouhcine et al. 2006).

In the assumption of case B recombination, two-thirds of the Lyman continuum photons are reprocessed as Ly $\alpha$. Therefor, young starbursts should be easily detected by their Ly $\alpha$ emission. However, observations of nearby starbursts (e.g. Hartmann et al. 1988; Calzetti \& Kinney 1992) revealed in most starburst galaxies a much weaker Ly $\alpha$ than predicted by simple models of galaxy formation. To explain this low flux, Charlot \& Fall (1993) suggested that Ly $\alpha$ strong emission requires either a low abundance of dust or an AGN. However, Giavalisco et al. (1996) demonstrated that pure extinction could not explain the weak Ly $\alpha$ fluxes. Results suggest that Ly $\alpha$ photons are decoupled from the continuum radiation resulting in a increased sensitivity to dust Neufeld (1990).

Ly $\alpha$ systems at $z>5$ are a very useful probe of the properties of very young star-forming systems at the epoch after reionization. Unfortunately, the data obtained to the date (e.g. Kodaira et al. 2003; Hu et al. 2004; Rhoads et al. 2004; Westra et al. 2005; Taniguchi et al. 2005; Ajiki et al. 2006a; Shimasaku et al. 2006) are not sufficient to place strong constraints on the fundamental properties. The Ly $\alpha$ line is not used commonly as a SFR tracer. Resonant scattering of $\operatorname{Ly} \alpha$ photons by neutral atomic hydrogen affects their relation with the SFR in a galaxy (Charlot \& Fall 1993).

Narrow-band imaging produces approximately volume-limited samples, since the narrowobserved bands correspond to small windows in redshift space. The objects are selected with a well defined limit in equivalent width and the line flux can be transformed into luminosity with some simple assumptions. Narrow-band imaging thus provide line luminosities for a volume-limited sample of emission line galaxies. In the case of detecting lines used as star formation tracers, the sample would be directly SFR-selected, except for the AGN contribution. The problem with this approach is that stars contaminating the sample or contributions from different emission lines cannot be separated only with narrow-band imaging. Additional assumptions (on the luminosity functions of ELGs, e.g. Jones \& Bland-Hawthorn 2001) or additional data (see, e.g., the color-color diagrams of Fujita et al. 2003) are needed to complete the scenario.

The aim of this work is to contribute to several aspects of the narrow-band imaging in order to optimize the results of the observations. Section 2 describes the characterization of the filters used through this work. 
In Section 3 we deal with the different sources of contamination, either galactic or extragalactic that can be found in an narrow-band survey and different methods used to detect them. Section 4 describes how to infer the line and continuum fluxes (and the corresponding equivalent width) from the measured magnitudes in a variety of scenarios, including more than one broad-band filter and more than one line inside the narrow-band filter. We study the method to select the candidates, based solely on broad and narrow-band colors in Sect. 5. We study in detail also the comoving volume covered by a narrow-band selected sample. Numeric examples with three filter set, including broad and narrow-band filters, are shown in Section 6. Although we have based our examples in two surveys carried out using filters at the $8200 \AA$ and $9200 \AA$ windows, the analysis and methods described in this work are valid for a wide variety of narrow-band imaging surveys, including those extended into the IR. Finally, in Sect. 7, we present helpful equations to compute the narrow-band and the broad-band exposure times in order to obtain minimum dispersion in the ratio of fluxes of both bands with minimum total exposure time. Conclusions are exposed in Sect. 8 .

\section{Characterization of the filters}

In general, the filters used in CCD photometry are characterized by their transmittance curve $T(\lambda)$. The fiducial transmittances are usually measured by the observatories with collimated beams and at room temperature. The total transmittance includes the contribution of the quantum efficiency (QE) of the detector and the transmittances of the optical systems in the light path. The working temperature, usually lower than the room temperature, produces a blue shift of the transmittance of the narrow band filters. A converging light beam passing through the filter produces a second blue shift, as well as a decrease of the overall transmittance and a broadening of the transmittance profile. These effects depend on the focal ratio of the beam passing through the filter.

Except in Section 5, where absolute source flux are needed for statistics, we use always the transmittance normalized to peak unity $(\mathcal{T})$ instead of the absolute transmittance $(T)$. Both are related by $T=T_{\text {peak }} \mathcal{T}$, where $T_{\text {peak }}$ is the maximum value of the transmittance.

The mean wavelength of the band, $\lambda_{0}$, is computed in agreement with the conventional definition (see for example Fukugita et al. 1995; Fiorucci \& Munari 2003).

$$
\lambda_{0} \equiv \frac{\int \lambda \mathcal{T}(\lambda) \mathrm{d} \lambda}{\int \mathcal{T}(\lambda) \mathrm{d} \lambda}
$$

$\Delta$ is the width of the filter computed from $\mathcal{T}$. It agrees with the definition of the equivalent 
width of the band transmittance profile $W_{0}$ of Fiorucci \& Munari (2003):

$$
W_{0} \equiv \Delta \equiv \int \mathcal{T}(\lambda) \mathrm{d} \lambda
$$

We use $\Delta$ instead of $W_{0}$ and reserve $W$ for the equivalent width of the emission lines.

$\mu^{2}$ (Eq. 7 of Fiorucci \& Munari 2003) is the second-order central momentum of the transmittance profile:

$$
\mu^{2} \equiv \frac{\int\left(\lambda-\lambda_{0}\right)^{2} \mathcal{T}(\lambda) \mathrm{d} \lambda}{\int \mathcal{T}(\lambda) \mathrm{d} \lambda}
$$

The flux calibration of the narrow-band images can be achieved using spectrophotometric standard stars, by obtaining synthetic magnitudes using the known SED of the spectrophotometric stars.

There are different choices for the election of the zero point of the narrow-band filter magnitude. The particular election does not affect the selection of objects or the measured fluxes. Different zero points in the bands only introduce an offset in the broad-narrow color.

One approach is to use AB magnitudes (Oke 1974) or ST magnitudes (J. Walsh 1995) 1 that provide a fixed physical magnitude scale. In other approximations, a broad-band filter is chosen as reference and the zero point of the narrow band is obtained by fixing a zero color for a particular SED. We adopt, unless noted otherwise, the normalization of color zero for the flat $f_{\lambda}$ spectrum. This is equivalent to make equal the zero points of both bands.

\section{Contaminating sources}

The redshift of each source entering the narrow-band filter is unknown a priori. Sources with strong emission lines (particularly, but not only, H $\alpha$, [OІІІ] $\lambda \lambda 4959,5007$, [OII] $\lambda 3727$ and Ly $\alpha$ ) produce a narrow-band flux excess. Different emission lines at different redshifts cannot be distinguished using only the narrow-band excess emission. Additionally, other sources (either galactic or extragalactic) without emission lines can exhibit a narrow-band flux excess when their SEDs passes through the filter set used for ELG selection. Galaxies and stars with abundant absorption features in the atmospheric windows studied can potentially appear in narrow-band surveys as emitting objects. This aspect has to be studied for each filter set particularly (in Section 6, examples with different filters are shown).

\footnotetext{
${ }^{1}$ Optical and UV spectrophotometric standard stars http://www . eso.org/observing/standards/spectra
} 
In general, stars show colors similar to those of the black body of their effective temperature. For the late spectral types, the presence of molecular bands change the trend and large colors can be observed. In the specific wavelength range of the two atmospheric windows, the absorption produced by some molecular species present in the atmosphere of cold stars (TiO, VO) dominates. For late K stars, broad-narrow colors diverge from the black body. White dwarfs with broad Hydrogen absorption lines would produce also a narrow-band flux depression.

It is important to note that depending on the filters used, particularly on the relative position of their $\lambda_{0}$, molecular bands of cold stars could produce an increase of the color. Those stars with a large color excess produced by absorption lines can be misclassified as ELGs. As the ratio of stars to galaxies decreases with increasing magnitude (see, for

example, Karaali et al. 2003), the number of contaminating stars do not dominate the candidate selection at faint magnitudes. In any case, particular care has to be taken in the design or election of the filter set in order to avoid this source of contamination.

Some types of stellar objects show emission lines in the wavelength interval where the filters are defined. Novae, symbiotic stars, cataclysmic variables (in general, low mass stars with either coronal activity or binaries) can show emission in the line Pa9 (9229 ^). After a nova explosion there is emission in the multiplet OI near 9261-9266 $\AA$ and the line HeII in $8236 \AA$. Lines of Ni and NaII between 8185 and $8242 \AA$ (Rudy et al. 2003) can also be found.

Massive stars, such as some Wolf-Rayet, can exhibit emission lines of Pa9 (9229 $\AA)$ and in HeII+CIV (8236 ̊) (Crowther et al. 2002).

The equivalent widths of the lines in the range covered by the narrow-band filters is of the order of tens of Angstroms. Although the relative frequency of appearance of these types of stars is very low, they can be also selected by their color excess.

\subsection{Classification schemes}

Further analysis is needed in other to classify the different objects selected by their narrow-band flux excess.

To separate the stars, we can apply morphological methods to the selected candidates. A example of that approach appears in McCracken et al. (2003). An alternative, and commonly used, approximation is to make use of the artificial neural network of the sourcecharacterization program SExtractor (Bertin \& Arnouts 1996). Nevertheless, these classifi- 
cation methods depend on the SNR and seeing of the images. It can be difficult to classify candidates morphologically when the overall SNR is low or the seeing is not optimal. Multicolor photometry can be also used to distinguish stars using a color criterion (we show a simple example in Sect. 4.3.1)

Methods to classify extragalactic sources range from statistical corrections (Jones \& Bland-Hawthorn 2001) to color-color criteria and photometric redshift determination. For example, Pascual et al. (2001) recomputed the statistical correction of Jones \& Bland-Hawthorn (2001) to find that the estimated contamination from galaxies at $z>0.24$ was negligible.

(Fujita et al. 2003) uses three photometric bands $\left(B, R_{C}\right.$ and $\left.I_{C}\right)$ and the GISSEL96 models (Bruzual \& Charlot 2003) to develop a color criterion that allow the authors to select $\mathrm{H} \alpha$ emitters at $z=0.24$. Following a similar procedure, but using spectra of emissionline galaxies rather than models, Ly et al. (2006) uses five photometric bands $\left(B, R_{C}, V, i^{\prime}\right.$ and $z^{\prime}$.

Ly $\alpha$ surveys use dropout technique in filters bluer than $i$ in order to assure that the Lyman break $(\lambda=912 \AA)$ lies bluewards the narrow-band filter. For example, Taniguchi et al. (2005) request that the candidates are not detected in $B, V$ and $R$, in addition to other criteria color criteria.

Finally, we mention a more complex classification scheme. It is presented in Wolf et al. (2001) and it is used in the CADIS survey (Hippelein et al. 2003). Twelve medium pass band filters are used, in addition to broad-band $B, R, I, J$ and $K^{\prime}$, to classify the objects in three broad categories: stars, quasars (including AGN) and galaxies.

\section{Measured flux and equivalent width of the emission line}

Fluxes of the objects through the filters are obtained integrating the spectrum of the object through the filter profile. The narrow-band filter technique allows, not only to select the objects with a possible emission line, but also to compute the value of the line flux and the equivalent width of the line.

In order to obtain the line and continuum flux for the selected objects, several simplifications, that are studied in this section, need to be made. First, the line profile is assumed infinitely thin when compared with the width of the narrow-band filter. Second, the positioning of the emission within the transmittance profile of the used filters is very close to the center of the narrow-band filter. With simple assumption we can obtain a mean wavelength for the emission line. Finally, the continuum flux can be modeled by an analytic function, 
either a power-law or a polynomial. It is critical to have a good estimate of the continuum flux.

\subsection{The infinitely thin line approximation}

The FWHM of the lines of star-forming galaxies is related with the mass of the object and typically is less than $\sim 10 \AA$. To estimate the influence of the finite width of the emission line we have integrated Gaussian profiles through Gaussian and rectangular narrow-band filters and computed the recovered line flux. The FWHM increases with redshift a factor $1+z$, so the infinitely thin line approximation can be valid, recovering more than $80 \%$ of the line flux, up to $z \sim 4$ for very narrow filters $(\Delta \sim 50 \AA)$. For wider filters, $(\Delta \sim 150 \AA)$, we recover more than $80 \%$ of the flux up to $z \sim 10$. These limit redshifts are lower for sources with broader lines, such as AGN. The ELGs can still be selected using the methods described in Sect. 5 but the fluxes of the lines computed with this section's equations will underestimate the total flux.

\subsection{Line flux and EW of a source with an infinitely thin line}

We assume that the SED of a source at redshift $z$ with a emission line of rest wavelength $\lambda_{r}$ can be expressed as the sum of a continuum contribution and an infinitely thin emission line with flux $f_{1}$. The emission line is represented analytically by a delta of Dirac centered in $\lambda_{z}=\lambda_{r}(1+z)$.

In the following we assume that we have identified the emission line detected inside the narrow-band filter. The exact wavelength of the line is also unknown, but it is located in a very narrow range inside the narrow-band filter. We handle the determination of an approximate redshift for the line in Sect. 4.2.2.

With the assumed conditions, the resulting SED is:

$$
f(\lambda)=f_{\mathrm{c}}(\lambda)+f_{1} \delta\left(\lambda-\lambda_{z}\right)
$$

with $f_{\mathrm{l}}$ constant and $f_{\mathrm{c}}(\lambda)$ an arbitrary function of the wavelength. Note that a negative value of $f_{1}$ produces an absorption line.

We define the mean flux through the filter as:

$$
\bar{f} \equiv \frac{\int f(\lambda) T(\lambda) \lambda \mathrm{d} \lambda}{\int T(\lambda) \lambda \mathrm{d} \lambda}=\frac{\int f(\lambda) \mathcal{T}(\lambda) \lambda \mathrm{d} \lambda}{\int \mathcal{T}(\lambda) \lambda \mathrm{d} \lambda}
$$


The mean flux of the SED of Eq. 4 is:

$$
\bar{f}=\bar{f}_{\mathrm{c}}+\left(\frac{\mathcal{T}\left(\lambda_{z}\right)}{\Delta}\right)\left(\frac{\lambda_{z}}{\lambda_{0}}\right) f_{\mathrm{l}}
$$

The mean flux is a function of the redshift of the source $z$ (or a function of $\lambda_{z}$, as both are related)2. The mean flux depends on the normalized transmittance of the filter at $\lambda_{z}$, the wavelength where the emission line is located and on the mean value of the continuum flux. We have not introduced yet any constraint to the shape of the continuum.

\subsubsection{Effective width of the filters}

Returning to Eq. 6, we introduce the effective width of the filter $\Delta^{\prime}$ :

$$
\Delta^{\prime}\left(\lambda_{z}\right) \equiv\left(\frac{\Delta}{\mathcal{T}\left(\lambda_{z}\right)}\right)\left(\frac{\lambda_{0}}{\lambda_{z}}\right)
$$

This definition is slightly different of the width obtained integrating the normalized transmittance. Both $\Delta$ and $\Delta^{\prime}$ are independent of the maximum transmittance of the filter. $\Delta^{\prime}$ depends on the redshift of the source.

The value of $\Delta^{\prime}$ is close to $\Delta$ in the center of the filter, in the region where the relative transmittance is close to unity. In the wings of the filter, the effective width increases and tends to infinity when the transmittance tends to zero. The physical meaning is that a emission line does not contribute to the integrated flux if it is positioned on a wavelength where the transmittance is near zero. The term in Eq 6 containing the line flux is effectively zero when the effective width tends to infinity.

The result of Waller (1990) is based on square filters. We can recover this result making the transmittance equal to unity and making $\lambda_{0}=\lambda_{z}$. In that case, the effective width of the filter reduces to:

$$
\Delta^{\prime}\left(\lambda_{z}\right)=\Delta\left(\frac{\lambda_{0}}{\lambda_{z}}\right) \simeq \Delta
$$

In general, the flux equation 6 can be rewritten finally as:

$$
\bar{f}=\bar{f}_{\mathrm{c}}+\frac{1}{\Delta^{\prime}\left(\lambda_{z}\right)} f_{\mathrm{l}}
$$

\footnotetext{
${ }^{2}$ We will use both variables interchangeably, as appropriated
} 


\subsubsection{Estimation of mean redshift}

We focus on the position of the emission line within the narrow-band filter. The definition of effective width depends on the unknown redshift of the source. As the candidates are selected by their color excess in a narrow-band filter, the object with emission lines will be distributed in a small range of wavelengths, given mainly by the transmittance of the narrow band filter. If $\mathrm{d} n$ is the number of galaxies with a emission line, whose rest wavelength is $\lambda_{r}$, the number of selected objects is $P\left(\lambda_{z}, m_{\mathrm{B}}, m_{\mathrm{N}}\right) \mathrm{d} n$. If we suppose that the probability of selecting a given object can be factorized in a term depending on $\lambda_{z}$ and in terms depending on the magnitudes, the mean value of an arbitrary function $h\left(\lambda_{z}\right)$ can be written as:

$$
\bar{h}=\frac{\int h\left(\lambda_{z}\right) p\left(\lambda_{z}\right) \mathrm{d} n}{\int p\left(\lambda_{z}\right) \mathrm{d} n}
$$

We can assume also that the probability $p\left(\lambda_{z}\right)$ is dominated by the shape of the narrowband filter and write:

$$
p\left(\lambda_{z}\right) \propto \Delta_{\mathrm{N}}^{\prime-1}
$$

Assuming a constant comoving density of galaxies inside the narrow-band filter, the number of galaxies is proportional to the comoving volume $V$ (for the explicit expression of the comoving volume, see Hogg 1999, and references therein). The final equation is:

$$
\bar{h}=\frac{\int h\left(\lambda_{z}\right) \Delta_{\mathrm{N}}^{\prime-1}\left(\lambda_{z}\right) \mathrm{d} n}{\int \Delta_{\mathrm{N}}^{\prime-1}\left(\lambda_{z}\right) \mathrm{d} n}=\frac{\int h\left(\lambda_{z}\right) \lambda_{z} \mathcal{T}_{\mathrm{N}}\left(\lambda_{z}\right) \mathrm{d} V(z)}{\int \lambda_{z} \mathcal{T}_{\mathrm{N}}\left(\lambda_{z}\right) \mathrm{d} V(z)}
$$

The mean value of $\lambda_{z}$ can be directly computed from last equation. The mean values computed with this method depend slightly on the cosmology parametrization selected (except on the value of the Hubble constant) and on the emission line chosen.

\subsection{Line flux and EW with different filter layouts}

The narrow-band filter layout used determines the approach used to approximate the continuum near the emission line. In a general case, we have $n$ filters and $n$ equations like Eq. 6, one for each filter. This provides us with, at most, $n-1$ parameters to parametrize the continuum and one parameter to characterize the line, $\left(f_{1}\right)$.

A power-law continuum $\left(f_{\mathrm{c}} \propto \lambda^{\beta}\right)$ can be fitted with at least three bands. It is even possible to assume a given power-law index (for example, the $\beta=-1$ for Ly $\alpha$ emitters in Ando et al. (2006)). Another possible functional form for the continuum is a polynomial of degree $n-2$. 
Most surveys use one narrow-band filter on the region of interest and one or more broad-band filters (e.g. Pascual et al. 2001; Hu et al. 2004; Ajiki et al. 2006a). Other surveys use more several contiguous narrow-band filters and a medium-band filter as a veto filter (Westra et al. 2006) or more complicated layouts (Hayes \& Ostlin 2006).

In the following sections we study two different cases of the polynomial functional form. With two broad-band filters, a linear dependency of the continuum flux with wavelength can be assumed. In the case of having one broad-band filter, the continuum has a constant value.

\subsubsection{Three filters and a linear continuum}

We develop here a general solution for the case of three filters covering the line. Solutions for other cases with more filters can be easily obtained.

The continuum is approximated by a linear term with slope $\gamma$. For simplicity, we select the parameters so that the linear term vanish at $\lambda_{z}$ :

$$
f_{\mathrm{c}}(\lambda)=f_{\mathrm{c}}\left(\lambda_{z}\right)+\gamma\left(\lambda-\lambda_{z}\right)
$$

Using Eq. 5, the effective flux is:

$$
\bar{f}=f_{\mathrm{c}}+\gamma\left(\lambda_{0}+\frac{\mu^{2}}{\lambda_{0}}-\lambda_{z}\right)+\frac{f_{1}}{\Delta^{\prime}\left(\lambda_{z}\right)}
$$

being $\mu$ the second order central moment of the transmittance of the filter (Eq. 3). We rename the factor of $\gamma$ as $s$ :

$$
s=\lambda_{0}+\frac{\mu^{2}}{\lambda_{0}}-\lambda_{z}
$$

We also substitute $\lambda_{z}$ by $\bar{\lambda}_{z}$ in $s$ and in $\Delta^{\prime}$. Equation 9 is written here for the three filters covering the line (denoted $\mathrm{A}, \mathrm{B}$ and $\mathrm{N}$ ).

$$
\begin{aligned}
& \bar{f}_{\mathrm{A}}=f_{\mathrm{c}}+\gamma s_{\mathrm{A}}+\frac{1}{\Delta_{\mathrm{A}}^{\prime}} f_{\mathrm{l}} \\
& \bar{f}_{\mathrm{B}}=f_{\mathrm{c}}+\gamma s_{\mathrm{B}}+\frac{1}{\Delta_{\mathrm{B}}^{\prime}} f_{1} \\
& \bar{f}_{\mathrm{N}}=f_{\mathrm{c}}+\gamma s_{\mathrm{N}}+\frac{1}{\Delta_{\mathrm{N}}^{\prime}} f_{1}
\end{aligned}
$$

This is a system of three linear equations with three unknowns $\left(f_{\mathrm{c}}, \gamma\right.$ and $\left.f_{1}\right)$ that can be resolved easily. 


\subsubsection{Two filters and a constant continuum}

In this case, we denote the filters by $\mathrm{B}$ and $\mathrm{N}$ and suppose that $\mathrm{N}$ is a narrow-band filter.

The fluxes inside the filters are:

$$
\bar{f}_{\mathrm{B}}=f_{\mathrm{c}}+\frac{1}{\Delta_{\mathrm{B}}^{\prime}} f_{\mathrm{l}} \quad \bar{f}_{\mathrm{N}}=f_{\mathrm{c}}+\frac{1}{\Delta_{\mathrm{N}}^{\prime}} f_{\mathrm{l}}
$$

We account for the different effective width of the filters introducing the new parameter $\epsilon \equiv \Delta_{\mathrm{N}}^{\prime} / \Delta_{\mathrm{B}}^{\prime}$. As $\mathrm{N}$ is a narrow-band filter, $\epsilon \ll 1$. The parameters of the emission line are as follows:

$$
\begin{aligned}
f_{\mathrm{l}} & =\Delta_{\mathrm{N}}^{\prime}\left(\bar{f}_{\mathrm{N}}-\bar{f}_{\mathrm{B}}\right) \frac{1}{1-\epsilon} \\
f_{\mathrm{c}} & =\bar{f}_{\mathrm{B}}\left(\frac{1-\epsilon \mathcal{Q}}{1-\epsilon}\right) \\
W & =\Delta_{\mathrm{N}}^{\prime}(\mathcal{Q}-1)\left(\frac{1}{1-\epsilon \mathcal{Q}}\right)
\end{aligned}
$$

being $\mathcal{Q} \equiv \bar{f}_{\mathrm{N}} / \bar{f}_{\mathrm{B}}$. The color of a source (with the color normalization of Sect. 2) is directly $m_{\mathrm{B}}-m_{\mathrm{N}}=2.5 \log \mathcal{Q}$.

Equation 22 shows that the EW does not change linearly with $\mathcal{Q}$. There exists an upper limit to the color that a source can have due to the presence of an emission line:

$$
\mathcal{Q}_{\max }=\lim _{W \rightarrow \infty} \mathcal{Q}(W)=\lim _{W \rightarrow \infty}\left(\frac{1+\frac{W}{\Delta_{\mathrm{N}}^{\prime}}}{1+\epsilon \frac{W}{\Delta_{\mathrm{N}}^{\prime}}}\right)=\frac{\Delta_{\mathrm{B}}^{\prime}}{\Delta_{\mathrm{N}}^{\prime}}
$$

It is worth noting that observing an emission-line object with a narrow-band filter increases the contrast between the emission line and the continuum, as the object is brighter in the line than in the continuum. Let us assume that we have two objects with the same magnitude in the broad-band, one with line of equivalent width $W$ (named L) and the other without emission (denoted $\mathrm{C}$ ). The ratio of the fluxes of the two objects measured in the narrow-band filter is the contrast parameter, defined originally by Thompson et al. (1995). Using Eq. 9, we obtain:

$$
\frac{f(\mathrm{~L})}{f(\mathrm{C})}=\frac{1+\frac{W}{\Delta_{\mathrm{N}}^{\prime}}}{1+\frac{W}{\Delta_{\mathrm{B}}^{\prime}}}
$$

In the original contrast parameter of Thompson et al. (1995), the width of the filter is characterized by its FWHM. For our Eq. 24 we, more accurately, use the effective width as 
defined in Eq. 7. The numeric expression of the contrast parameter is equal to the equation obtained by inverting Eq. 22,

There are two limit cases for these equations. When the width of the broad-band is much greater than the narrow-band width, we can consider that the line do not contribute to the broad-band filter. The parameter $\epsilon$ tends to zero and the equations can be written as:

$$
\begin{aligned}
f_{1} & =\Delta_{\mathrm{N}}^{\prime}\left(\bar{f}_{\mathrm{N}}-\bar{f}_{\mathrm{B}}\right) \\
f_{\mathrm{c}} & =\bar{f}_{\mathrm{B}} \\
W & =\Delta_{\mathrm{N}}^{\prime}\left(\frac{\bar{f}_{\mathrm{N}}}{\bar{f}_{\mathrm{B}}}-1\right)=\Delta_{\mathrm{N}}^{\prime}(\mathcal{Q}-1)
\end{aligned}
$$

The second limit case is when one filter do not cover the emission line. Thus, the line do not contribute to the flux in that band and the final equations are again Eqs. 25- 27.

\subsection{Multiple lines inside the filter}

In most cases of astrophysical interest, more than one line lies inside the narrow-band filter. For example, $\mathrm{H} \alpha$ plus [NII] $\lambda \lambda 6548,6584$, and [OIII] $\lambda \lambda 4959,5007$ plus $\mathrm{H} \beta$. The flux recovered from the narrow-band filter comes from all the lines, although each line experiences a different filter transmittance.

We write Eq. [4 with three different lines (the case of $\mathrm{H} \alpha$ and $[\mathrm{OIII}] \lambda \lambda 4959,5007$ ) and denote their fluxes and wavelength with super index 1,2 and 3:

$$
f(\lambda)=f_{\mathrm{c}}+f_{1}^{1} \delta\left(\lambda-\lambda_{z}^{1}\right)+f_{1}^{2} \delta\left(\lambda-\lambda_{z}^{2}\right)+f_{1}^{3} \delta\left(\lambda-\lambda_{z}^{3}\right)
$$

and Eq. 9 is substituted by:

$$
\bar{f}=f_{\mathrm{c}}+\frac{1}{\Delta^{\prime}\left(\lambda_{z}^{1}\right)} f_{1}^{1}+\frac{1}{\Delta^{\prime}\left(\lambda_{z}^{2}\right)} f_{1}^{2}+\frac{1}{\Delta^{\prime}\left(\lambda_{z}^{3}\right)} f_{1}^{3}
$$

The wavelengths of the three lines can be obtained from the redshift (or from the wavelength) of one of them (for example, $\lambda_{z}^{2}$ and $\lambda_{z}^{3}$ as a function of $\lambda_{z}^{1}$ ). We suppose that line 1 is the line we are interested on.

If the emission lines fluxes have known ratios, we can simplify the equation:

$$
\bar{f}=f_{\mathrm{c}}+f_{1}^{1}\left(\frac{1}{\Delta^{\prime}\left(\lambda_{z}^{1}\right)}+\frac{1}{\Delta^{\prime}\left(\lambda_{z}^{2}\right)} \frac{f_{1}^{2}}{f_{1}^{1}}+\frac{1}{\Delta^{\prime}\left(\lambda_{z}^{3}\right)} \frac{f_{1}^{3}}{f_{1}^{1}}\right)
$$


The effective width of the filter is substituted by a combined width $\left(\Delta^{\prime \prime}\right)$ that depends on the particular set of lines studied and their ratios, that we denote $r_{2}=\frac{f_{1}^{2}}{f_{1}^{1}}$ and $r_{3}=\frac{f_{1}^{3}}{f_{1}^{1}}$. The combined width is:

$$
\Delta^{\prime \prime}\left(\lambda_{z}^{1}, r_{2}, r_{3}\right) \equiv\left(\frac{1}{\Delta^{\prime}\left(\lambda_{z}^{1}\right)}+\frac{1}{\Delta^{\prime}\left(\lambda_{z}^{2}\right)} r_{2}+\frac{1}{\Delta^{\prime}\left(\lambda_{z}^{3}\right)} r_{3}\right)^{-1}
$$

The equation developed previously to obtain fluxes and EW are valid to obtain directly the flux of the main line (line with index 1) using $\Delta^{\prime \prime}$ instead of $\Delta^{\prime}$. The mean wavelength $\overline{\lambda_{z}}$ is altered also. It is still computed using Eq. [12, but substituting $\Delta^{\prime}$ by $\Delta^{\prime \prime}$.

The ratio of the combined width with the width computed in the single line case represents the fraction of the total flux that comes from the main line. We denote this ratio by $\phi$.

$$
\phi\left(\lambda_{z}^{1}, r_{2}, r_{3}\right)=\frac{\Delta^{\prime \prime}\left(\lambda_{z}^{1}, r_{2}, r_{3}\right)}{\Delta^{\prime}\left(\lambda_{z}^{1}\right)}
$$

The fraction $\phi$ depends on the shape of the filter and the different line ratios of the lines. We can obtain a limit case of $\phi$ when the filters are wide (this is the case for broad-band filters). With that assumption, $\Delta^{\prime}\left(\lambda_{z}^{1}\right) \simeq \Delta^{\prime}\left(\lambda_{z}^{2}\right) \simeq \Delta^{\prime}\left(\lambda_{z}^{3}\right)$ and Eq. 32 can be written as:

$$
\phi_{l}\left(r_{2}, r_{3}\right)=\left(1+r_{2}+r_{3}\right)^{-1}
$$

In the following subsections we apply Eq. 31 - 33 for the particular cases of $\mathrm{H} \alpha$ and [OIII].

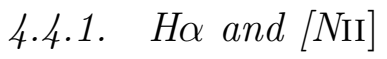

Both lines of the doublet [NII] are separated about $20 \AA$ from $\mathrm{H} \alpha$ in the rest frame. In this case, $\lambda_{z}^{1}$ is the redshifted wavelength of $\mathrm{H} \alpha, \lambda_{z}^{2}$ is the redshift wavelength of [NII] $\lambda_{6548}$ and $\lambda_{z}^{3}$ is the redshifted wavelength of $[\mathrm{NII}] \lambda 6584$.

The global ratio I([NII])/I(H $\alpha)$ is related with metallicity (van Zee et al. 1998; Melbourne \& Salzer 2002; Kewlev \& Dopita 2002; Pettini \& Pagel 2004) and AGN activity (Veilleux \& Osterbrock 1987; Swinbank et al. 2004), showing a great range of variation between star-forming galaxies.

Historically, $\mathrm{H} \alpha$ surveys that could not separate [NII] from $\mathrm{H} \alpha$ have used mean values to correct for the contribution of $[\mathrm{NII}]$. A commonly assumed mean value of the flux ratio $\mathrm{I}([\mathrm{NII}] \lambda 6584) / \mathrm{I}(\mathrm{H} \alpha)$ is 0.32 . This is the value reported by Kennicutt (1992) and the UCM survey (Gallego et al. 1997) (James et al. 2005, see also). 
This particular mean value is widely used in studies of the SFR based on $\mathrm{H} \alpha$, where the lines of [NII] can not be resolved (e.g. Tresse \& Maddox 1998; Yan et al. 1999; Iwamuro et al. 2000; Pascual et al. 2001; Fujita et al. 2003; Umeda et al. 2004).

The same line ratio is obtained from the Sloan Digital Sky Survey Data Release 43 (SDSS DR4, Adelman-McCarthy et al. 2006) if we apply a lower cutoff in EW of $20 \AA$ (the detection limit of the UCM survey), typical of classical emission-line galaxy surveys.

In Fig. 1$]$ we show the ratio $\mathrm{I}([\mathrm{NII}]) / \mathrm{I}(\mathrm{H} \alpha)$ represented as a function of the equivalent width of $\mathrm{H} \alpha$ for the galaxies of the UCM survey and the SDSS. The equivalent width represented is observed, not rest frame. As the redshift of the main galaxy sample of SDSS is at most $z \sim 0.1$, the uncertainty produced by not correcting is about $4 \%$. The effect in the $\mathrm{UCM}$ is even less, as the galaxies in the sample are local.

The mean value assumed is represented as a straight line. We can see the wide range of variation of $\mathrm{I}([\mathrm{NII}]) / \mathrm{I}(\mathrm{H} \alpha)$, from near 0.02 to 4 . There is a tendency of decreasing $\mathrm{I}([\mathrm{NII}]) / \mathrm{I}(\mathrm{H} \alpha)$ with increasing $\mathrm{EW}$, corresponding to a decrease of the metallicity. 1989).

The intensity of the nitrogen lines is related: $\mathrm{I}([\mathrm{NII}] \lambda 6584) / \mathrm{I}([\mathrm{NII}] \lambda 6548)=3$ (Osterbrock

We can write Eq. 31 as a function of $\mathrm{r}=\mathrm{I}([\mathrm{NII}] \lambda 6584) / \mathrm{I}(\mathrm{H} \alpha)$ only. In this case, the combined effective width of the filter is:

$$
\Delta^{\prime \prime}\left(\lambda_{z}^{1}, r\right)=\left(\frac{1}{\Delta^{\prime}\left(\lambda_{z}^{1}\right)}+r\left(\frac{1}{3 \Delta^{\prime}\left(\lambda_{z}^{2}\right)}+\frac{1}{\Delta^{\prime}\left(\lambda_{z}^{3}\right)}\right)\right)^{-1}
$$

We obtain the mean redshift of the sources using Eq. 12 and inserting there the $\Delta^{\prime \prime}\left(\lambda_{z}, r\right)$. We can obtain also the limit parameter $\phi_{l}=(1+4 / 3 r)^{-1}$. With the mean value assumed of $r=0.32, \phi_{l}=0.71$. This means that, in the ideal case of a square filter covering the three lines and the given line ratios, $71 \%$ of the flux recovered in Eq. 20 comes from $\mathrm{H} \alpha$ and the rest comes from [NII].

A narrow-band filter with the same $\phi$ value than the broad-band filter is wide enough to contain simultaneously the two nitrogen lines and $\mathrm{H} \alpha$. A greater value of $\phi$ implies that the $[\mathrm{NII}]$ lines only enter partially inside the narrow line filter. Using the standard $[\mathrm{NII}] \lambda \lambda 6548,6584$ correction with very narrow filters produce a systematic under estimation of the line flux.

We can further refine the $[\mathrm{NII}] \lambda \lambda 6548,6584$ correction. From Fig 1 we can obtain a

\footnotetext{
${ }^{3}$ http://www.mpa-garching.mpg.de/SDSS/DR4/
} 
relation between the equivalent width $W(\mathrm{H} \alpha)$ and $r=\mathrm{I}([\mathrm{NII}] \lambda \lambda 6548,6584) / \mathrm{I}(\mathrm{H} \alpha)$. Using that relation we can iterate through Eq. 22 and obtain simultaneously $r$ and EW. With this procedure, every object has a different value of $r$, which implies different values of $\Delta^{\prime \prime}$ and $\phi$ for every object.

\subsection{2. $H \beta$ and [OIII]}

In this case, three lines enter the filter, the doublet [OIII] and $\mathrm{H} \beta . \mathrm{H} \beta$ is separated $146 \AA$ from $[\mathrm{OIII}] \lambda 5007$ in the rest frame. We can write Eq. 31 with $\lambda_{z}^{3}$ as the redshifted wavelength of $\mathrm{H} \beta, \lambda_{z}^{2}$ is the redshift wavelength of [OIII] $\lambda 4959$ and $\lambda_{z}^{1}$ is the redshifted wavelength of [ÖII] $\lambda 5007$.

In Fig 2 we show the ratio [OIII] $\lambda 5007 / \mathrm{H} \beta$ again for galaxies of the SDSS DR4 and for galaxies of the UCM sample. We have only represented galaxies with the lines [OIII] $\lambda \lambda 4959,5007$, $\mathrm{H} \beta$ and a $\mathrm{EW}(\mathrm{H} \alpha)>20 \AA$, in order to mimic the limit values of classical ELG surveys. The mean value obtained for $\mathrm{I}(\mathrm{H} \beta) / \mathrm{I}([\mathrm{OIII}] \lambda 5007)$ in the SDSS is $r=1.05$ (the result for the $\mathrm{UCM}$ is compatible, $r=1.1$ ).

We write Eq. 31 for this particular case as a function of $r=\mathrm{I}(\mathrm{H} \beta) / \mathrm{I}([\mathrm{OIII}] \lambda 5007)$ and knowing that I([OIII $] \lambda 5007) / \mathrm{I}([\mathrm{OIII}] \lambda 4959)=3$ (Osterbrock 1989):

$$
\Delta^{\prime \prime}\left(\lambda_{z}^{1}, r\right)=\left(\frac{1}{\Delta^{\prime}\left(\lambda_{z}^{1}\right)}+\frac{1}{3 \Delta^{\prime}\left(\lambda_{z}^{2}\right)}+r \frac{1}{\Delta^{\prime}\left(\lambda_{z}^{3}\right)}\right)^{-1}
$$

The limit parameter is in this case $\phi_{l}=(4 / 3+r)^{-1}$. With the mean value assumed for $r, \phi_{l}=0.42$, meaning that only $42 \%$ of the flux would come from [OIII] $\lambda 5007$.

In this case, $\phi$ is very close to unity for the narrow-band filters. This means that [OIII] $\lambda 5007$ will enter alone in the filter. In the case of broad-band, the ratio is close to the limit value, meaning that the three lines enter the broad-band filter. As a summary, we can consider that [OII] $\lambda 5007$ enters alone in the narrow-band filter, but we have to include the three lines when considering the broad-band filter.

\subsection{Line luminosity of the objects}

The line luminosity of each object can be calculated from the measured line flux and the redshift of the object (obtained from $\bar{\lambda}_{z}$ ). The line flux corrected from obscuration by 


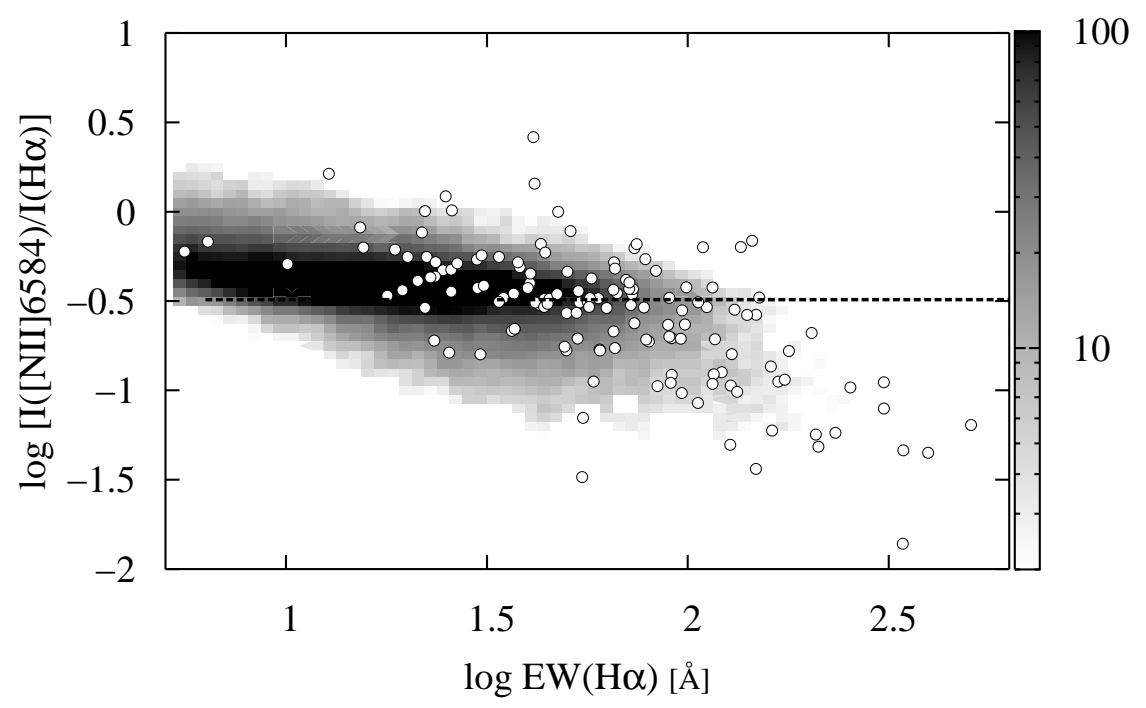

Fig. 1.- Ratio I([NII] $\lambda 6584) / \mathrm{I}(\mathrm{H} \alpha)$ represented as a function of the equivalent width of $\mathrm{H} \alpha$ for the galaxies of the UCM survey (white circles) and the Sloan Digital Sky Survey (the number of galaxies is represented in gray scale). The mean value assumed in this work is represented as a straight line.

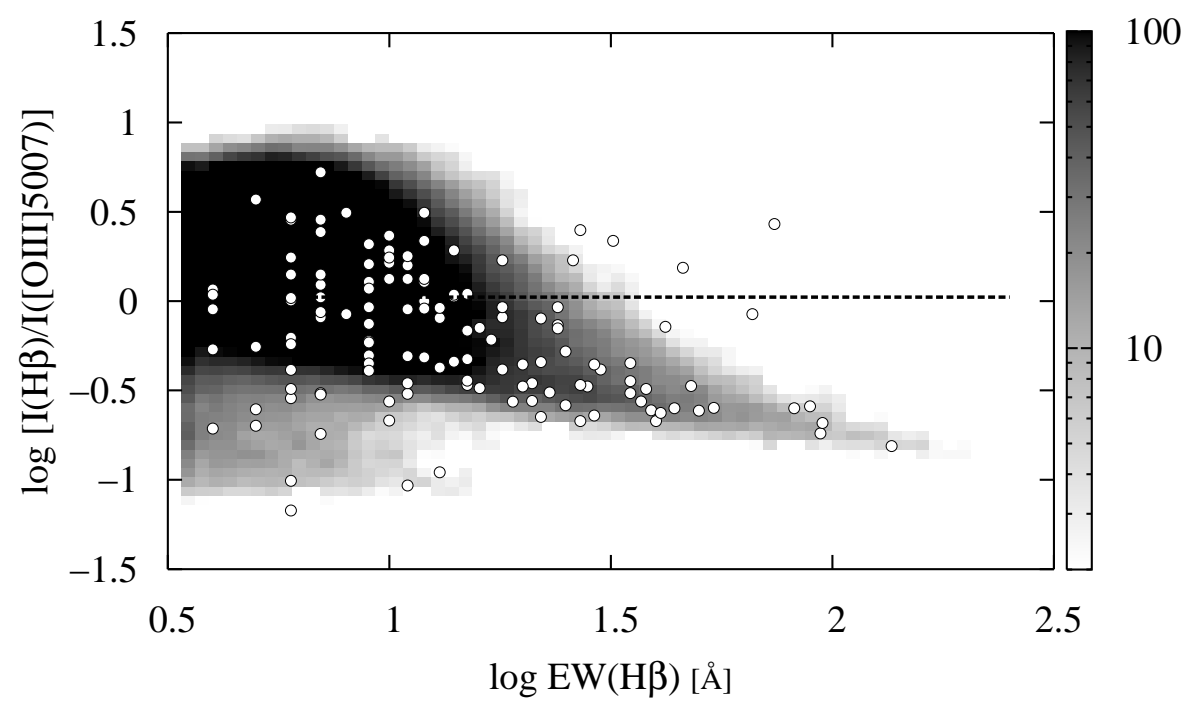

Fig. 2.- Ratio $\mathrm{I}(\mathrm{H} \beta) / \mathrm{I}([\mathrm{OIII}] \lambda 5007)$ represented as a function of the equivalent width of $\mathrm{H} \beta$ for the galaxies of the UCM survey (white circles) and the Sloan Digital Sky Survey (the number of galaxies is represented in gray scale). The mean value assumed in this work is represented as a straight line. 
dust $\left(f_{1}^{0}\right)$ can be obtained from the measured line flux $\left(f_{1}\right)$ :

$$
\log f_{1}^{0}=\log f_{1}+0.4\left(A_{\lambda_{r}}+a_{\lambda_{z}}\right)
$$

being $A_{\lambda_{r}}$ the internal obscuration at the rest frame wavelength $\lambda_{r}$ and $a_{\lambda_{z}}$ the galactic obscuration. The line luminosity is then:

$$
L=4 \pi d_{\mathrm{L}}^{2}(\bar{z}) f_{\mathrm{l}}^{0}
$$

being $d_{\mathrm{L}}(z)$ the luminosity distance.

The common obscuration corrections applied in narrow-band searches are discussed in the following subsection.

\subsubsection{The obscuration correction}

Obscuration by dust affects seriously any measurement of galaxy luminosities at the optical and UV wavelengths. Correcting for this effect is a difficult task as measurements of parameters sensitive to obscuration are not always easy to obtain.

In the case of obscuration corrections for emission lines, it is common to assume an average value of $1^{m}$ in $\mathrm{H} \alpha$ and the extinction curve of Cardelli et al. (1989) to apply the value to other emission lines. This value of $A_{H \alpha}$ has been found as the average of the obscuration of several samples of local $\mathrm{H} \alpha$ emitting galaxies (e.g. Kennicutt 1983; Gallego et al. 1995; Hopkins et al. 2003). This procedure has the advantage that is very simple to apply to a luminosity function or luminosity density, since it is a scaling factor.

It is worth to note here that the average obscuration applied in UV studies is about $A_{V}=1^{m}$ (Tresse et al. 1996; Sullivan et al. 2000, e.g.). In the GALEX far-ultraviolet (FUV), Buat et al. (2005) found a mean obscuration of $A_{F U V}=1.1^{m}$ for galaxies selected in the GALEX near-ultraviolet band. This value is roughly $A_{V} \simeq 0.4^{m}$. Galaxies in the GALEX UV Imaging Atlas of Nearby Galaxies (Gil de Paz et al. 2004, 2006) have a mean obscuration, considering only spirals and irregulars, of $A_{F U V}=1.8^{m}$, corresponding to $A_{V} \simeq 0.7^{m}$ (Gil de Paz, private communication) as this sample can be considered as selected in the optical.

The average obscuration of $A_{H \alpha}=1^{m}$ corresponds to a value of about $A_{V} \simeq 1.22^{m}$ as noted by Hopkins (2004). This disagreement in the obscuration is a selection effect of the different (FUV, UV and $\mathrm{H} \alpha$ ) samples. Galaxies selected at redder wavelengths can have greater internal obscurations than those selected at the UV, where heavily absorbed systems will not be selected. 
The value of $A$ at the wavelength of the emission line is obtained applying a given extinction curve. We are assuming that the continuum and the emission line are affected by the same obscuration, so the EW does not need obscuration correction. This last assumption is not true if we use the obscuration recipe of Calzetti et al. (1996), that is applied for strong starbursts. However, normal galaxies and moderate starbursts can be assumed as showing no obscuration correction in EW.

\section{Magnitude-color selection criterion}

In this Section, we study the underlying statistics of the magnitude-color diagram used to select ELGs in the most simple scenario, a broad-band filter and a narrow-band filter. A sample selected in broad-narrow color is directly selected in EW (Eq. 22). Thus, ideally, a sample can be created selecting the objects with color in excess of a minimum color $\left(m_{\mathrm{B}}-m_{\mathrm{N}}\right)_{\min }$ that translates into a minimum equivalent width $\mathrm{EW}_{\min }$.

The dispersion in the magnitude-color diagram is dominated by the uncertainties in the magnitudes in the faint region and by the different colors of the objects in the bright region. Furthermore, the mean value of the color, $\mu\left[m_{\mathrm{B}}-m_{\mathrm{N}}\right]$, depends on $m_{\mathrm{N}}$, because different types of objects can dominate the color at different narrow-band magnitude ranges. The method of selection has to account for the different sources of dispersion of the color. A solution is to compute the standard deviation of the color dispersion and consider candidates, with a level of signification $n_{\sigma}$, the objects with color:

$$
m_{\mathrm{B}}-m_{\mathrm{N}}>\max \left\{\left(m_{\mathrm{B}}-m_{\mathrm{N}}\right)_{\min }, \mu\left[m_{\mathrm{B}}-m_{\mathrm{N}}\right]+n_{\sigma} \sigma\left[m_{\mathrm{B}}-m_{\mathrm{N}}\right]\right\}
$$

The standard deviation of the color distribution, as a function of the narrow-band magnitude, can be calculated from the distribution of objects. In the following subsection, we infer an analytic expression that can be useful when there are not enough points in the data to calculate directly the dispersion.

\subsection{Standard deviation of the color distribution}

Let us assume a population of objects with the same SED, given by $f(\lambda)$, so all the objects have the same color.

In general, the source flux in analog to digital units (ADU) collected in a CCD detector from an object are proportional to the integrated flux of the source through the pass band 
and the exposure time $t$ :

$$
N \propto t \int f(\lambda) T(\lambda) \lambda \mathrm{d} \lambda
$$

Equation 39] can be written in terms of $\bar{f}, \lambda_{0}$ and $\Delta$ as follows:

$$
N \propto t \bar{f} T_{\text {peak }} \Delta \lambda_{0}
$$

where $T_{\text {peak }}$ is the maximum value of the transmittance.

The ratio $\mathcal{Q}=\bar{f}_{\mathrm{N}} / \bar{f}_{\mathrm{B}}$ is proportional to the ratio of ADUs in the same bands $\mathcal{R}$ :

$$
\mathcal{R} \equiv \frac{N_{\mathrm{N}}}{N_{\mathrm{B}}}=\mathcal{Q}\left(\frac{\lambda_{0}^{\mathrm{N}}}{\lambda_{0}^{\mathrm{B}}}\right)\left(\frac{\Delta_{\mathrm{N}}}{\Delta_{\mathrm{B}}}\right)\left(\frac{T_{\text {peak }}^{\mathrm{N}}}{T_{\text {peak }}^{\mathrm{B}}}\right)\left(\frac{t_{\mathrm{N}}}{t_{\mathrm{B}}}\right)=b \mathcal{Q}\left(\frac{t_{\mathrm{N}}}{t_{\mathrm{B}}}\right)
$$

In general, $\mathcal{Q}$ is a function with a strong dependency on the wavelength because of night sky emission lines. In the cases covered in the present work, the filters are selected to exclude strong emission features in the sky spectrum but that is not always possible, such as when using narrow band IR filters designed to cover emission lines of astrophysical interest at zero redshift like $\operatorname{Br} \gamma$ or molecular hydrogen.

We include the parameters depending of the transmittance of the filters in:

$$
b \equiv\left(\frac{\lambda_{0}^{\mathrm{N}}}{\lambda_{0}^{\mathrm{B}}}\right)\left(\frac{T_{\text {peak }}^{\mathrm{N}}}{T_{\text {peak }}^{\mathrm{B}}}\right)\left(\frac{\Delta_{\mathrm{N}}}{\Delta_{\mathrm{B}}}\right)
$$

Eq. 41 applies independently of the flux source. We denote the quantities referred to the sky background with a index $S$ and the quantities referred to a particular object by $O$. In particular:

$$
\mathcal{R}_{\mathrm{S}}=\frac{N_{\mathrm{N}}^{\mathrm{S}}}{N_{\mathrm{B}}^{\mathrm{S}}}=b \mathcal{Q}_{\mathrm{S}}\left(\frac{t_{\mathrm{N}}}{t_{\mathrm{B}}}\right)
$$

The standard deviation of $\mathcal{Q}_{\mathrm{O}}$ can be obtained from the standard deviation of the ratio of the source flux in ADUs: $\mathcal{R}_{\mathrm{O}}$ as:

$$
\left(\frac{\sigma\left[\mathcal{Q}_{\mathrm{O}}\right]}{\mathcal{Q}_{\mathrm{O}}}\right)^{2}=\left(\frac{\sigma\left[\mathcal{R}_{\mathrm{O}}\right]}{\mathcal{R}_{\mathrm{O}}}\right)^{2}=\left(\frac{\sigma\left[N_{\mathrm{B}}^{\mathrm{O}}\right]}{N_{\mathrm{B}}^{\mathrm{O}}}\right)^{2}+\left(\frac{\sigma\left[N_{\mathrm{N}}^{\mathrm{O}}\right]}{N_{\mathrm{N}}^{\mathrm{O}}}\right)^{2}
$$

${ }^{4}$ Equation 40 can be also written in terms of $\lambda_{\text {eff }}$ (Fiorucci \& Munari 2003, Eq. 24) as follows:

$$
N \propto t \tilde{f} T_{\text {peak }} \Delta \lambda_{\text {eff }}
$$

being $\tilde{f}=\frac{\int f(\lambda) \mathcal{T}(\lambda) \mathrm{d} \lambda}{\int \mathcal{T}(\lambda) \mathrm{d} \lambda}$ and $\lambda_{\text {eff }}=\frac{\int \lambda f(\lambda) \mathcal{T}(\lambda) \mathrm{d} \lambda}{\int f(\lambda) \mathcal{T}(\lambda) \mathrm{d} \lambda}$

We prefer using $\lambda_{0}$ to avoid the flux dependence of $\lambda_{\text {eff }}$, that would complicate the equations of Section 4 . 
We do not include a covariance term. The covariance between the sources flux in ADUs should be negligible because we are measuring different parts of the spectrum with very different filters.

The terms in the right-hand side of the equation are the inverses of the signal-noise ratio (SNR) We adopt here the signal to noise ratio as computed by Howell et al. (1996) with a slightly changed notation:

$$
\mathrm{SNR} \equiv \frac{N_{\mathrm{O}}}{\sigma\left[N_{\mathrm{O}}\right]}=\frac{N_{\mathrm{O}}}{\sqrt{G\left(N_{\mathrm{O}}+A n_{\mathrm{S}}\right)+A G^{2} R^{2}}}
$$

$N_{\mathrm{O}}$ are the ADUs of the object in a given band, $n_{\mathrm{S}}$ the best estimation of the sky background per pixel, $A$ the area in pixels of the aperture used to measure photometry, $R$ is the readout noise in ADUs and $G$ the inverse of the gain $g$. The term involving the readout noise can be neglected when the exposures are long enough to be background limited. so the dominating contribution to the noise are the Poisson terms. Note that the background limit can be difficult to reach if the narrow-band filter is particularly narrow, as the airglow windows considered in the present work are regions of low sky background.

Equation 45 depends on the ADUs of the object and on the sky background measured in both bands. We use Eq. 41 to translate the flux in the broad band into flux in the narrow band, assuming a given color for the object $\left(\mathcal{Q}_{\mathrm{O}}\right)$ and for the sky background $\left(\mathcal{Q}_{\mathrm{S}}\right)$. Finally, we put the equation as a function of the ADUs per unit time and pixel of the sky $\left(\dot{n}_{\mathrm{N}}^{\mathrm{S}}\right)$ and of the ADUs per unit time of the object $\left(\dot{N}_{\mathrm{N}}^{\mathrm{O}}\right)$, so we can easily operate in magnitudes:

$$
\begin{aligned}
\left(\frac{\sigma\left[\mathcal{Q}_{\mathrm{O}}\right]}{\mathcal{Q}_{\mathrm{O}}}\right)^{2} & =G\left(\frac{1}{t_{\mathrm{N}}}+b \mathcal{Q}_{\mathrm{O}} \frac{1}{t_{\mathrm{B}}}\right) \frac{1}{\dot{N}_{\mathrm{N}}^{\mathrm{O}}}+ \\
& +G q_{\mathrm{N}}\left(\frac{1}{t_{\mathrm{N}}}+b \frac{\mathcal{Q}_{\mathrm{O}}^{2}}{\mathcal{Q}_{\mathrm{S}}} \frac{1}{t_{\mathrm{B}}}\right) \frac{1}{\dot{N}_{\mathrm{N}}^{\mathrm{O}}}+ \\
& +A G^{2} R^{2}\left(\frac{1}{t_{\mathrm{N}}^{2}}+b^{2} \mathcal{Q}_{\mathrm{O}}^{2} \frac{1}{t_{\mathrm{B}}^{2}}\right)\left(\frac{1}{\dot{N}_{\mathrm{N}}^{\mathrm{O}}}\right)^{2}
\end{aligned}
$$

We introduce a new parameter $q$ that indicates if we are in the noise regime dominated by the background or in the regime dominated by the Poisson noise produced exclusively by the source flux. The parameter $q$ is the ratio between the flux in ADUs (or ADUs per unit time) coming from the sky background and that coming from the object, inside the given aperture $A$ and measured with a certain filter:

$$
q \equiv A \frac{n_{\mathrm{S}}}{N_{\mathrm{O}}}=A \frac{\dot{n}_{\mathrm{S}}}{\dot{N}_{\mathrm{O}}}=\frac{A}{p^{2}} 10^{-0.4(\mu-m)}
$$


where $\mu$ is the surface brightness of the sky, $m$ the magnitude of the object and $p$ the plate scale. $q$ tends to zero when the background level is low compared with the brightness of the object and to infinity when the background dominates over the object.

The previous equation is written in terms of $q_{\mathrm{N}}$, but can be rewritten easily in terms of $q_{\mathrm{B}}$, as $q_{\mathrm{N}}=q_{\mathrm{B}} \mathcal{Q}_{\mathrm{S}} / \mathcal{Q}_{\mathrm{O}}$.

Equation 46 shows $\sigma\left[\mathcal{Q}_{\mathrm{O}}\right]$ as a function of $\dot{N}_{\mathrm{N}}^{\mathrm{O}}$, and through it, as a function of the narrow-band magnitude. Of the three terms of the equation, the last corresponds to the readout noise and is negligible except for very short exposure. The second term comes from the contribution of the sky background and dominates for faint objects. The first term is produced by the ADUs of the object and only is important (when compared with the sky contribution) for bright objects.

The presence of a logarithm in the transformation between flux and magnitude produces an asymmetric distribution of points around the mean color, mainly in the region of faint objects. We obtain this behavior by defining two curves

$$
\begin{aligned}
& \left(m_{\mathrm{B}}-m_{\mathrm{N}}\right)_{-n_{\sigma}}=2.5 \log \left(\mathcal{Q}-n_{\sigma} \sigma[\mathcal{Q}]\right) \\
& \left(m_{\mathrm{B}}-m_{\mathrm{N}}\right)_{+n_{\sigma}}=2.5 \log \left(\mathcal{Q}+n_{\sigma} \sigma[\mathcal{Q}]\right)
\end{aligned}
$$

with $n_{\sigma}$ the level over the mean.

As can be seen from Eq. 46, for a given narrow-band magnitude, $\sigma\left[\mathcal{Q}_{\mathrm{O}}\right]$ depends on the properties of the filters encoded in $b$, on the exposure times in the two bands $\left(t_{\mathrm{N}}\right.$ and $\left.t_{\mathrm{B}}\right)$, on the color of the sky background $\left(\mathcal{Q}_{\mathrm{S}}\right)$ and on the relative brightness of the sky when compared with the brightness of the objects $(q)$. In Fig. 3 we show how the selection curve changes (with a fixed value of $n_{\sigma}=3$ ) with the exposure time and sky background in the two bands for the sample filter set CAFOS8200 (the different filter properties are detailed in Sect. 6) The increase of exposure time or the reduction of the sky background (effectively increasing the sky-background magnitude) reduces the slope of the selection curve.

\subsection{Isolines in the magnitude-color space}

Isolines represent curves joining points with an equal given property. Lines with equal EW correspond with lines of equal color in the magnitude-color diagram, but lines with equal continuum or line flux are not directly related with neither the color nor the magnitude of any of the bands. 


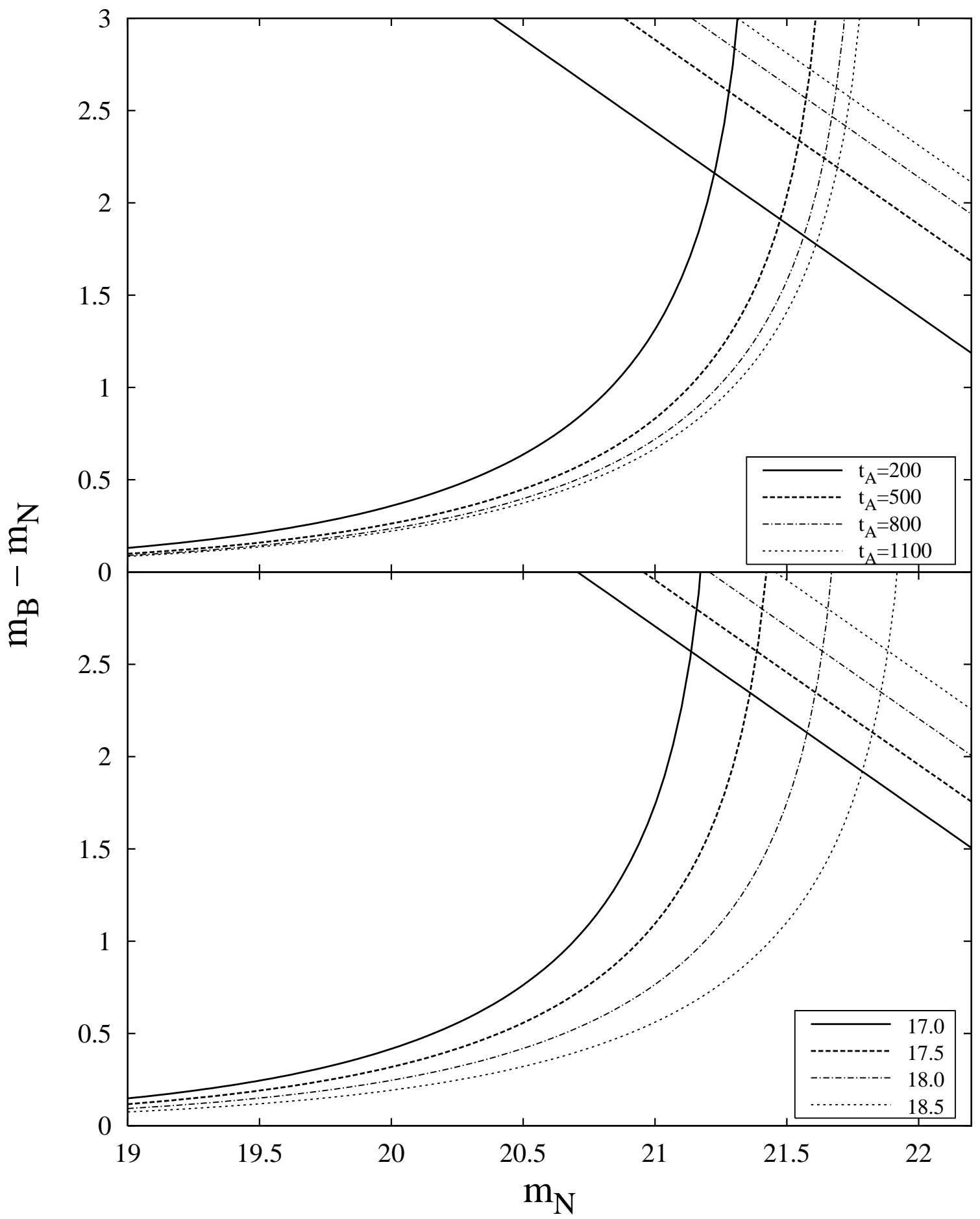

Fig. 3.- Selection curves with several different exposure times (top) and sky background (bottom). The broad-band exposure times are: 200, 500, 800 and $1100 \mathrm{~s}$, whereas the exposure time in the narrow-band filter is $800 \mathrm{~s}$. The sky background broad-band magnitudes are $17^{m}, 17.5^{m}, 18^{m}$ and $18.5^{m}$. We represent also the 5 - $\sigma$ broad-band limit magnitude (lines crossing on right top) corresponding to each exposure time and sky background magnitude. 


\subsubsection{Isolines of line flux}

We can compute the isolines in magnitude-color space starting with Eq. 9. If we assume a constant line flux $f_{1}$, the magnitudes of the objects as a function of $f_{1}$ and EW only are:

$$
\begin{aligned}
m_{\mathrm{B}} & =C-2.5 \log \left[f_{1}\left(\frac{1}{W}+\frac{1}{\Delta_{\mathrm{B}}^{\prime}}\right)\right] \\
m_{\mathrm{N}} & =C-2.5 \log \left[f_{1}\left(\frac{1}{W}+\frac{1}{\Delta_{\mathrm{N}}^{\prime}}\right)\right] \\
m_{\mathrm{B}}-m_{\mathrm{N}} & =2.5 \log \left(\frac{1+\frac{W}{\Delta_{\mathrm{N}}^{\prime}}}{1+\frac{W}{\Delta_{\mathrm{B}}^{\prime}}}\right)
\end{aligned}
$$

The luminosity of the emission line is proportional to the measured flux (see Sect. 4.5 for details), so the isolines of line flux are also isolines of line luminosity.

We can write a parametric expression for the isolines of line flux. We use $w=W / \Delta_{\mathrm{N}}^{\prime}$ as an adimensional parameter. Note that the equations are also valid for absorption lines $\left(f_{1}<0\right.$ and $W<0$ ). To simplify the notation, we rename the color $m_{\mathrm{B}}-m_{\mathrm{N}}$ as the independent variable $y$ and the narrow-band magnitude as $x$.

The parametric equations of the line flux isolines are:

$$
\begin{aligned}
& x=x_{\infty}-2.5 \log \left|1+\frac{1}{w}\right| \\
& y=2.5 \log \left(\frac{1+w}{1+\epsilon w}\right)
\end{aligned}
$$

where $x_{\infty}$ is the narrow-band magnitude of an object dominated by the emission line: $x_{\infty}=C-2.5 \log \left|\frac{f_{1}}{\Delta_{\mathrm{N}}^{\prime}}\right|$. Equations 53 and 54 are valid in the ranges of emission lines, corresponding to $w \in(0, \infty)$, and in the domain of absorptions lines, where the adimensional parameter $w \in(-1,0)$.

The asymptotic behavior of the curve is as follows. For an object without line $w \rightarrow 0$; the color $y \rightarrow 0$. When $w$ increases asymptotically the object is dominated by the emission line. Then , $x \rightarrow x_{\infty}$ (the magnitude of an object dominated by the emission line) and the color $y \rightarrow-2.5 \log \epsilon$. When $w \rightarrow-1$, the object is dominated by the absorption line; the absorption line grows stronger and the narrow-band magnitude tends to positive infinity; the object is progressively dimmer as the absorption line increases its equivalent width. The broad-narrow color tends to negative infinity. 


\subsubsection{Isolines of continuum flux}

The isolines of continuum flux can also be obtained:

$$
\begin{aligned}
& m_{\mathrm{B}}=C-2.5 \log \left[f_{\mathrm{c}}\left(1+\frac{W}{\Delta_{\mathrm{B}}^{\prime}}\right)\right] \\
& m_{\mathrm{N}}=C-2.5 \log \left[f_{\mathrm{c}}\left(1+\frac{W}{\Delta_{\mathrm{N}}^{\prime}}\right)\right]
\end{aligned}
$$

The equation of the color $m_{\mathrm{B}}-m_{\mathrm{N}}$ is equal to Eq. 52, because the color depends only on the equivalent width. A similar approach to obtain the equation of the isolines of continuum flux can be used. The adimensional parameter is again $w=W / \Delta_{\mathrm{N}}^{\prime}$ and the parametric equations are:

$$
\begin{aligned}
& x=x_{0}-2.5 \log (1+w) \\
& y=2.5 \log \left(\frac{1+w}{1+\epsilon w}\right)
\end{aligned}
$$

being $x_{0}=C-2.5 \log \left(f_{\mathrm{c}}\right)$ the magnitude on an object without line and $w \in(-1, \infty)$. The asymptotic limits correspond to a object without line $\left(w \rightarrow 0, x \rightarrow x_{0}\right.$ and $\left.y \rightarrow 0\right)$, an object dominated by the emission line $(w \rightarrow \infty, x \rightarrow-\infty$ and $y \rightarrow-2.5 \log \epsilon)$ and an object dominated by an absorption line $(w \rightarrow-1, x \rightarrow \infty$ and $y \rightarrow-\infty)$

In Fig. 4, we show a magnitude-color selection diagram including example isolines for

the WFC9200 case (see Sect. 6 for details). The isolines of continuum flux and line flux are represented by the dashed and solid curves respectively. We can see clearly the asymptotic behavior of both types of isolines. Although the limited numeric precision of the plot do not show it, the curves of line flux continue up to the maximum color.

\subsection{Redshift range of detectability and covered volume}

We have assigned an approximate redshift $\bar{z}$ to the source, but we do not know which is the redshift range $z_{1} z_{2}$ of the objects that could be selected and included in the sample. The answer to this question is the key for the incompleteness correction. If we intend to compute a luminosity function, we need to compute the comoving volume per object.

We consider that the object is at redshift $\bar{z}$ and it has a line flux $f_{1}$ and continuum flux $f_{\mathrm{c}}$. The flux recovered in a given filter at redshift $z$ (whereby the emission line lies at wavelength $\lambda_{z}$ ) is:

$$
f(z)=\left(f_{\mathrm{c}}\left(\frac{1+\bar{z}}{1+z}\right)+\frac{f_{\mathrm{l}}}{\Delta^{\prime}\left(\lambda_{z}\right)}\right)\left(\frac{d_{L}(\bar{z})}{d_{L}(z)}\right)^{2}
$$




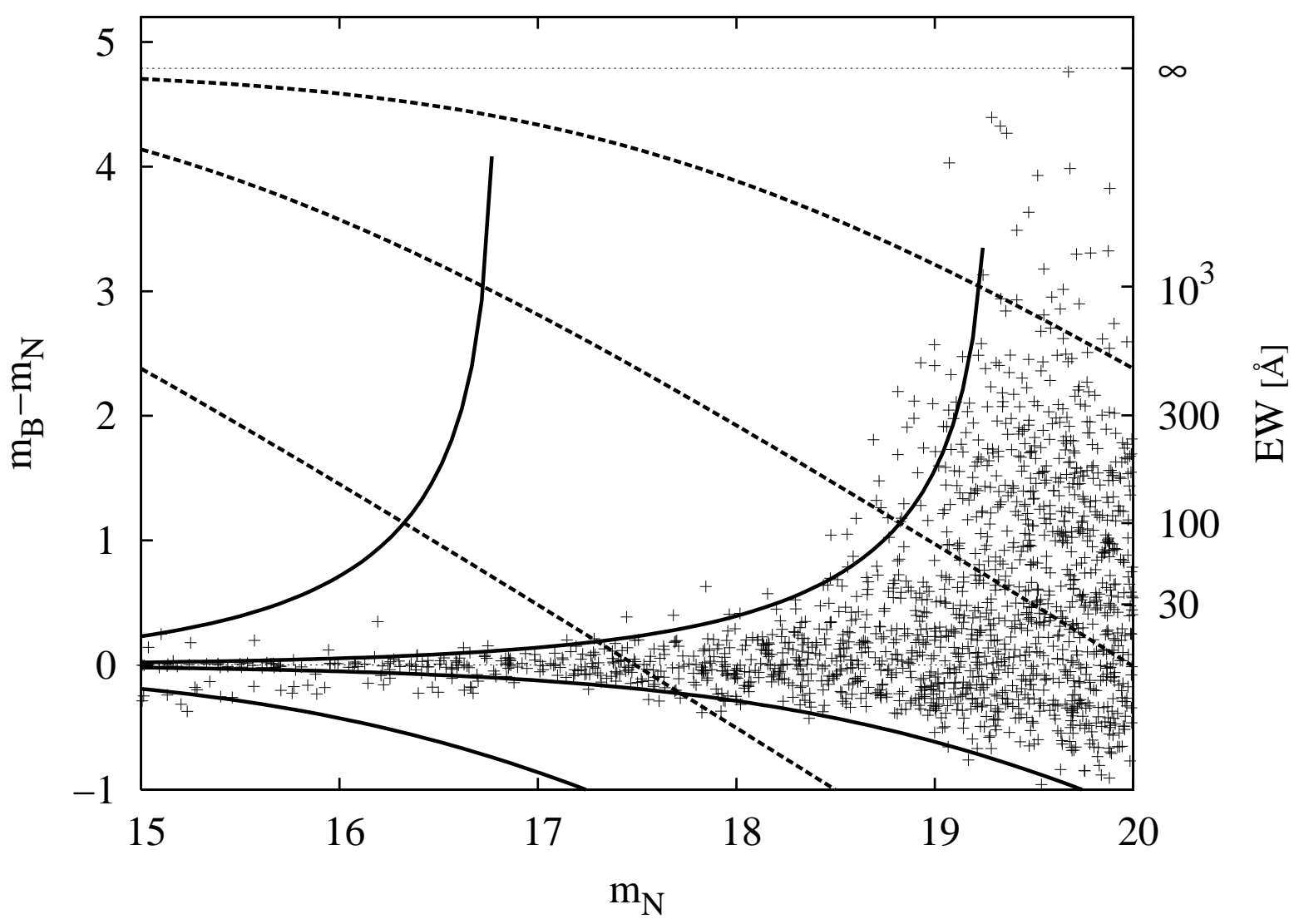

Fig. 4.- Isolines of line flux (solid curve) and continuum flux (dashed curve) for emission lines (broad-narrow color above zero) and absorption lines (bellow zero) in images obtained with the WFC9200 system (see Sect. 6 for details). The flux lines correspond to $10^{-14}$ and $10^{-15} \mathrm{erg} \mathrm{s}^{-1}$. The continuum lines are $10^{-16}, 10^{-17}$ and $10^{-18} \mathrm{erg} \mathrm{s}^{-1} \AA^{-1}$. 
with $d_{L}$ the luminosity distance. For $z=\bar{z}$ we recover Eq. 9. Substituting the appropriate filter widths $\Delta^{\prime}$, we can obtain the magnitude and color of the object as a function of redshift. Only at a range of redshifts $z_{1}, z_{2}$ the object fulfills the color criterion given in Sect. 5 ,

In Fig. 5 we show the redshift traces given by Eq. 59 for two objects in a magnitude-color diagram corresponding to the filter set CAFOS8200 (see Sect. 6 for details). The position of the objects in the diagram at redshift $\bar{z}$ are represented as crosses on top of the traces. The intersection between the traces and the selection curve (drawn with a dashed line) is labeled as $z_{1}$ and $z_{2}$. The object would be selected only in the range $z_{1}<z<z_{2}$.

The values of $z_{1}$ and $z_{2}$ depend through Eq. [59, on the transmittance of the filters. As the transmittance of the broad-band filter is mainly constant in the wavelength range of interest, the narrow-band filter dominates. Thus, the redshift range $z_{1}, z_{2}$ is small and dominated by the shape of the transmittance of the narrow-band filter. The redshift range depends also on the selection curve. Objects selected at lower signification level are closer to the selection limit and the redshift range $z_{1}, z_{2}$ is smaller.

The comoving volume where the object could be selected is computed from the redshifts $z_{1}, z_{2}$. This volume is one of the ingredients needed to compute the luminosity function. With Eq. 59 and the selection curve, we can compute a comoving volume for each object. It can be seen clearly that, with the same EW, faint objects have smaller comoving volumes. These objects have, for a fixed EW, low line flux (and low line luminosity). Thus, assuming a global comoving volume for all the detected objects tends to underestimate the value of the luminosity function for the faint objects and objects selected near the selection curve.

\section{Example filters}

\subsection{Sets of filters and instruments}

In order to provide observational tests for our analysis, we select three sets of filters in two different telescopes. The first instrument is CAFOS on the $2.2 \mathrm{~m}$ telescope at Calar Alto Observatory (Almería, Spain). The filters used were 816/16 (narrow band) and 850/150c (broad band, Johnson I). This is the instrumental setup used in the Pascual et al. (2001) survey. The second instrument is the Wide Field Camera (WFC, Ives et al. 1996), located at the Isaac Newton Telescope in the Observatorio del Roque de los Muchachos (island of La Palma, Spain). Two different narrow-band filters have been used, \#209 and \#208, together with the broad band filter \#194 (RGO I).

The transmittance of the different filters is shown in Fig. 6. The fiducial transmittances 


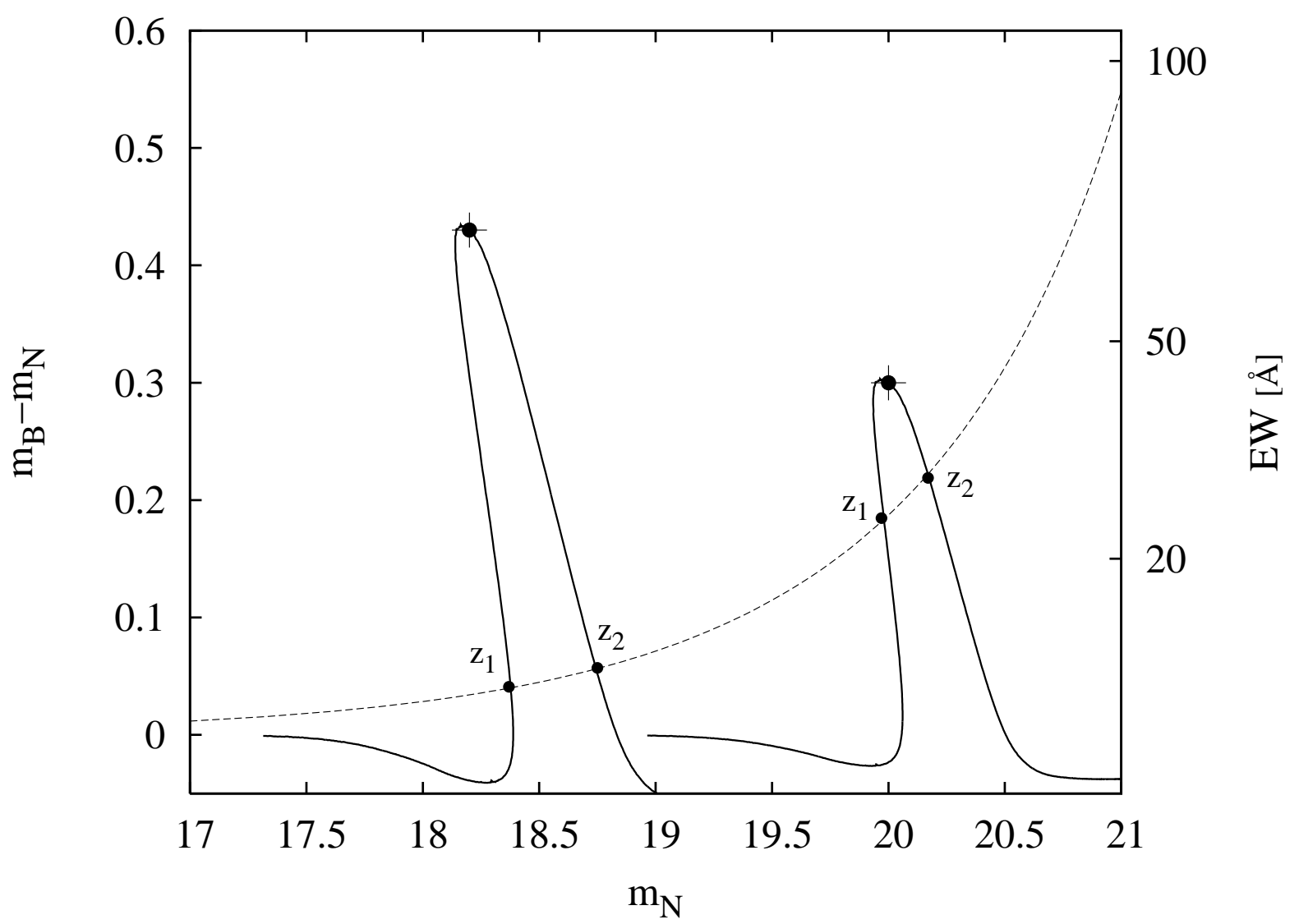

Fig. 5.- Redshift traces (solid lines) of two objects marked as crosses in a magnitude-color diagram for CAFOS8200 (see Sect. 6 for details). The emission line in this case is H $\alpha$. The selection limit is the dashed line. The intersection between the traces and the selection curve delimits the redshift range (from $z_{1}$ to $z_{2}$ in the plot) where the object could still be selected. 
are displayed together with the QE of the detector and the total transmittance. For a working temperature of $0^{\circ} \mathrm{C}$, the blue shift produced by the temperature and the converging beam for CAFOS 8200 is $10 \AA$, about $5 \%$ of the width of the filter (S. Pedraz, private communication). The blue shift is $17 \AA$ for the filter WFC8200 and $19 \AA$ for the filter WFC9200 (King et al. 1994). These two shifts are about $\sim 35 \%$ of the width of the filters. The converging beam can produce also an effect in the absolute transmittances and widths, but it turned out to be negligible for our examples.

Several quantities useful to describe filters are summarized in Table 1. Columns 1 and 2 are the name of the filter set and the name of the filter (either broad- or narrow-band). In the next three columns we show $\lambda_{0}($ column 3$), \sqrt{\mu^{2}}$ (column 4) and $\Delta$ (column 5) computed directly from the transmittance. Column 6 is the estimated mean value of $\lambda_{z}$ computed with Eq. 12, concordance cosmology and the $\mathrm{H} \alpha$ line, not including the corrections for the [NII] lines (see bellow). With $\bar{\lambda}_{z}$ we compute the effective width of the filter in column 7 . The difference between commonly used cosmologies and different emission lines is less than $5 \%$ in all cases. Note that the filter \#194, when used together with \#208 has an effective width roughly three times its width. The wavelength where the emission line is located has low transmittance. In this case, using $\Delta$ instead of $\Delta^{\prime}$ would underestimate the continuum flux.

In Table 2, we summarize the mean values of the combined width and $\phi$ parameter (Eq 32 for $\mathrm{H} \alpha$ and [OIII]-computed quantities with concordance cosmology. Columns 1 and 2 have the same meaning in Table 1. Columns 3 to 5 correspond to H $\alpha$-computed quantities with $r=\mathrm{I}([\mathrm{NII}] \lambda 6584) / \mathrm{I}(\mathrm{H} \alpha)=0.32$ (Sect. 4.4.1) and columns 6 to 8 to [OIII] with $r=\mathrm{I}(\mathrm{H} \beta) / \mathrm{I}([\mathrm{OIII}] \lambda 5007)=1.05$ (Sect 4.4.2). Columns 3 and 6 are the mean wavelengths computed according to Eq 12, using the value of $\Delta^{\prime \prime}$. Columns 4 and 7 the value of the combined width at the computed mean wavelength value and columns 5 and 8 the parameter $\phi$, that denotes the the fraction of the total flux that comes from the main line.

For $\mathrm{H} \alpha$, the narrow-band filter in the CAFOS8200 filter set has the same $\phi$ value than the broad-band filters. This means that is wide enough to contain simultaneously the two nitrogen lines and $\mathrm{H} \alpha$. The narrow-band filters of WFC8200 and WFC9200 have a greater $\phi$, meaning that the $[\mathrm{NII}]$ lines only enter partially inside the narrow line filter. In this two last cases, $82 \%$ and $85 \%$ of the flux in the narrow-band filter come from $\mathrm{H} \alpha$, instead of the nominal $71 \%$. Using the standard $[\mathrm{NII}] \lambda \lambda 6548,6584$ correction with very narrow filters such as the narrow-band filters of WFC, produce a systematic under estimation of the line flux of about $15 \%$.

In the case of [OIII], $\phi$ is very close to unity for the narrow-band filters. This means that $[\mathrm{OIII}] \lambda 5007$ will enter alone in the filter. In the case of broad-band, the ratio is close to the limit value ( 0.42 with the assumed value of $r$ ), meaning that the three lines enter 


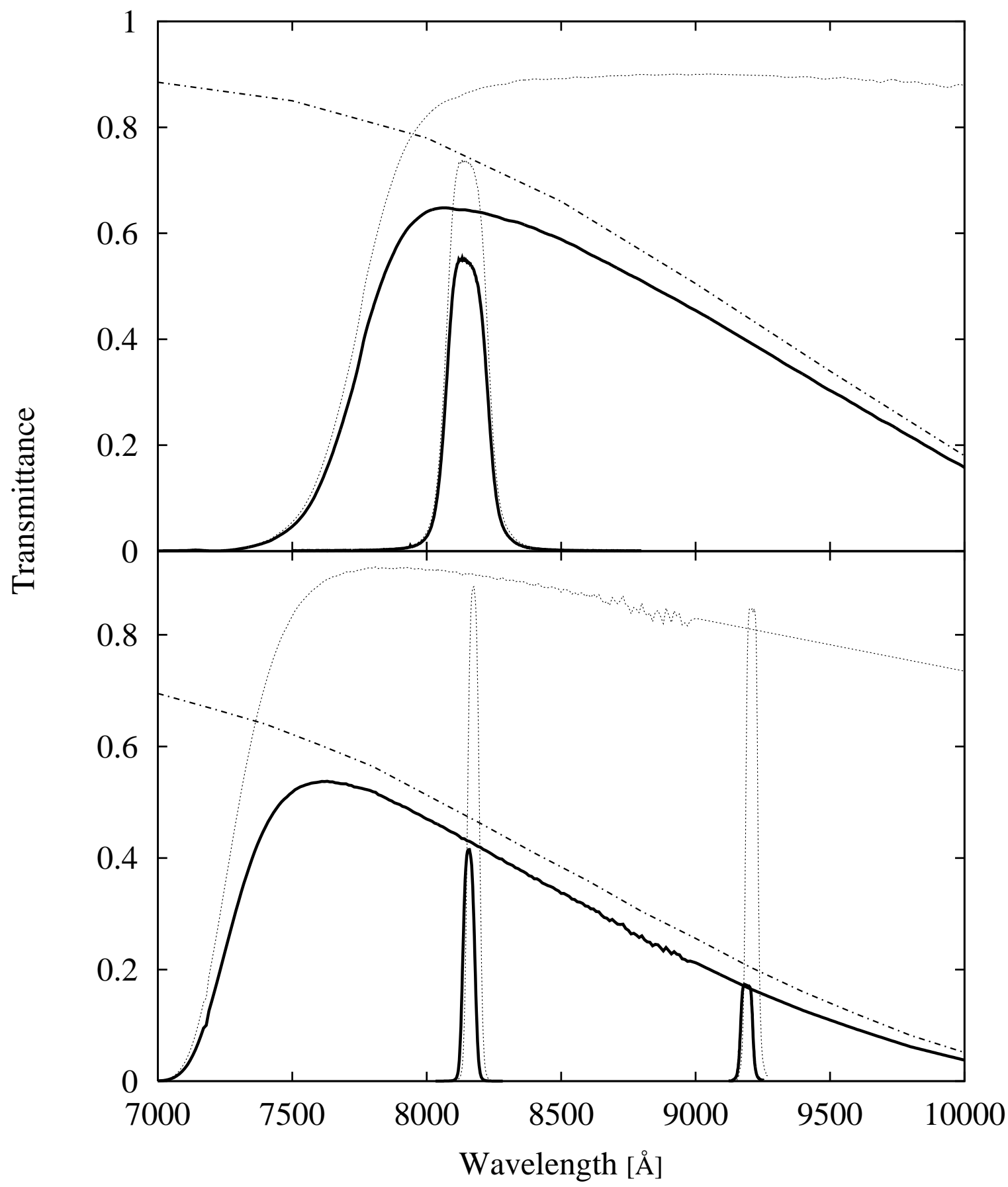

Fig. 6.- Transmittance curve for the different sets of filters. Dash-dotted lines represent the quantum efficiency, dotted lines represent the fiducial transmittance of the filters. Finally, the solid lines represent the real transmittance corrected from the different contributions detailed in the text. On top, the filters from CAFOS. Broad-band is $850 / 150 \mathrm{c}$ and narrowband 816/16. On bottom the filters from WFC. Broad-band is \#194 and narrow-band are \#209 around $8200 \AA$ and \#208 around $9200 \AA$. 
the broad-band filter. As a summary, we can consider that [OIII] $\lambda 5007$ enters alone in the narrow-band filter, but we have to include the three lines in the broad-band filter.

\subsection{Colors of galaxies}

We use galaxy templates to calculate the color evolution of different galaxies with increasing redshift. With the set of templates of Kinney et al. (1996), we have obtained colors for the three groups of sample filters. The templates have a resolution of $5 \AA$. We compare three wide types of galaxies with similar colors in this particular system: ellipticals and bulges, early-type spirals are represented by a Sb and finally late-type spirals and starbursts are represented by the starburst template SB1. The Sa and Sb spectra contain the $\mathrm{H} \alpha$ line in emission. The Sc and the starburst spectra also contain the nebular lines [OII] $\lambda 3727, \mathrm{H} \beta$ and $[\mathrm{OIII}] \lambda \lambda 4959,5007$.

We show the color $m_{\mathrm{B}}-m_{\mathrm{N}}$ of the different templates as a function of redshift up to $z=2$ in Fig. 7 for the three filter sets. Only the effect of the redshift is considered. We are not including galactic evolution. As expected, galaxies with different emission lines show strong increases on their colors when the emission lines enter the region covered by the filter. In the filter set WFC9200, elliptical templates exhibit color excess at redshifts around $z \sim 1$ and higher. This excess is not produced by emission lines. Instead, the very different central wavelengths of the two filters involved and the slope of the continuum near the $4000 \AA$ break explains the broad-narrow color obtained.

An additional color can help to distinguish different types of objects with narrow-band color excess. Figure 8 shows a $I$ - NB versus $I-Z$ color-color diagram for the WFC8200 filter set. We represents simultaneously stars, the black-body and galaxy templates. With a $I$ - NB versus NB diagram we can not distinguish between the color excess produced by the emission line of a starburst galaxy and by the molecular bands of a late-type star. But these objects have different $I-Z$ color. Late type stars are very red, $I-Z>0.4$ and starbursts are blue, with $I-Z<0.4$. Thus, we can classify objects using their $I-Z$ color.

\subsection{Colors of stars}

Using the library of Pickles (1998)5 we have estimated the colors of stars of different spectral types in our sample sets. The library contains spectral energy distributions of stars

\footnotetext{
${ }^{5}$ http://www.ifa.hawaii.edu/users/pickles/AJP/hilib.html
} 
Table 1. Summary of properties of the filters used

\begin{tabular}{|c|c|c|c|c|c|c|}
\hline System & $\begin{array}{l}\text { Filter } \\
(2)\end{array}$ & $\begin{array}{c}\lambda_{0} \\
(\AA) \\
(3)\end{array}$ & $\begin{array}{l}\sqrt{\mu^{2}} \\
(\AA) \\
(4)\end{array}$ & $\begin{array}{l}\Delta \\
(\AA) \\
(5)\end{array}$ & $\begin{array}{c}\bar{\lambda}_{z} \\
(\AA) \\
(6)\end{array}$ & $\begin{array}{c}\Delta^{\prime}\left(\bar{\lambda}_{z}\right) \\
(\AA) \\
(7)\end{array}$ \\
\hline \multirow[t]{2}{*}{ CAFOS8200 } & 816/16 (narrow) & 8139 & 80 & 173 & 8147 & 176 \\
\hline & 850/150c (broad) & 8715 & 643 & 1665 & 8147 & 1783 \\
\hline \multirow[t]{2}{*}{ WFC8200 } & \#209 (narrow) & 8157 & 17 & 48 & 8157 & 48 \\
\hline & \#194 (broad) & 8007 & 477 & 1333 & 8157 & 1940 \\
\hline \multirow[t]{2}{*}{ WFC9200 } & \#208 (narrow) & 9190 & 17 & 49 & 9190 & 49 \\
\hline & \#194 (broad) & 8007 & 477 & 1333 & 9190 & 4398 \\
\hline
\end{tabular}

Table 2. Mean combined effective widths of the filters

\begin{tabular}{|c|c|c|c|c|c|c|c|}
\hline \multirow{3}{*}{$\begin{array}{c}\text { System } \\
\text { (1) }\end{array}$} & \multirow{3}{*}{$\begin{array}{l}\text { Filter } \\
(2)\end{array}$} & \multicolumn{3}{|c|}{$\mathrm{H} \alpha+[\mathrm{NII}]$ with $r=0.32$} & \multicolumn{3}{|c|}{$\mathrm{H} \beta+[\mathrm{OIII}]$ with $r=1.05$} \\
\hline & & $\begin{array}{c}\bar{\lambda}_{z} \\
(\AA)\end{array}$ & $\begin{array}{c}\Delta^{\prime \prime}\left(\overline{\lambda_{z}}\right) \\
(\AA)\end{array}$ & $\phi$ & $\begin{array}{l}\bar{\lambda}_{z} \\
(\AA)\end{array}$ & $\begin{array}{c}\Delta^{\prime \prime}\left(\overline{\lambda_{z}}\right) \\
(\AA)\end{array}$ & $\phi$ \\
\hline & & $(3)$ & $(4)$ & (5) & (6) & $(7)$ & (8) \\
\hline \multirow[t]{2}{*}{ CAFOS 8200} & 816/16 (narrow) & 8142 & 123.5 & 0.70 & 8154 & 140.1 & 0.80 \\
\hline & 850/150c (broad) & 8142 & 1262 & 0.71 & 8154 & 763 & 0.43 \\
\hline \multirow[t]{2}{*}{ WFC8200 } & \#209 (narrow) & 8153 & 39.3 & 0.82 & 8157 & 47.5 & 0.99 \\
\hline & \#194 (broad) & 8153 & 1375 & 0.71 & 8157 & 779 & 0.40 \\
\hline \multirow[t]{2}{*}{ WFC9200 } & \#208 (narrow) & 9185 & 43.4 & 0.89 & 9190 & 45.8 & 0.93 \\
\hline & \#194 (broad) & 9185 & 3109 & 0.71 & 9190 & 1583 & 0.36 \\
\hline
\end{tabular}




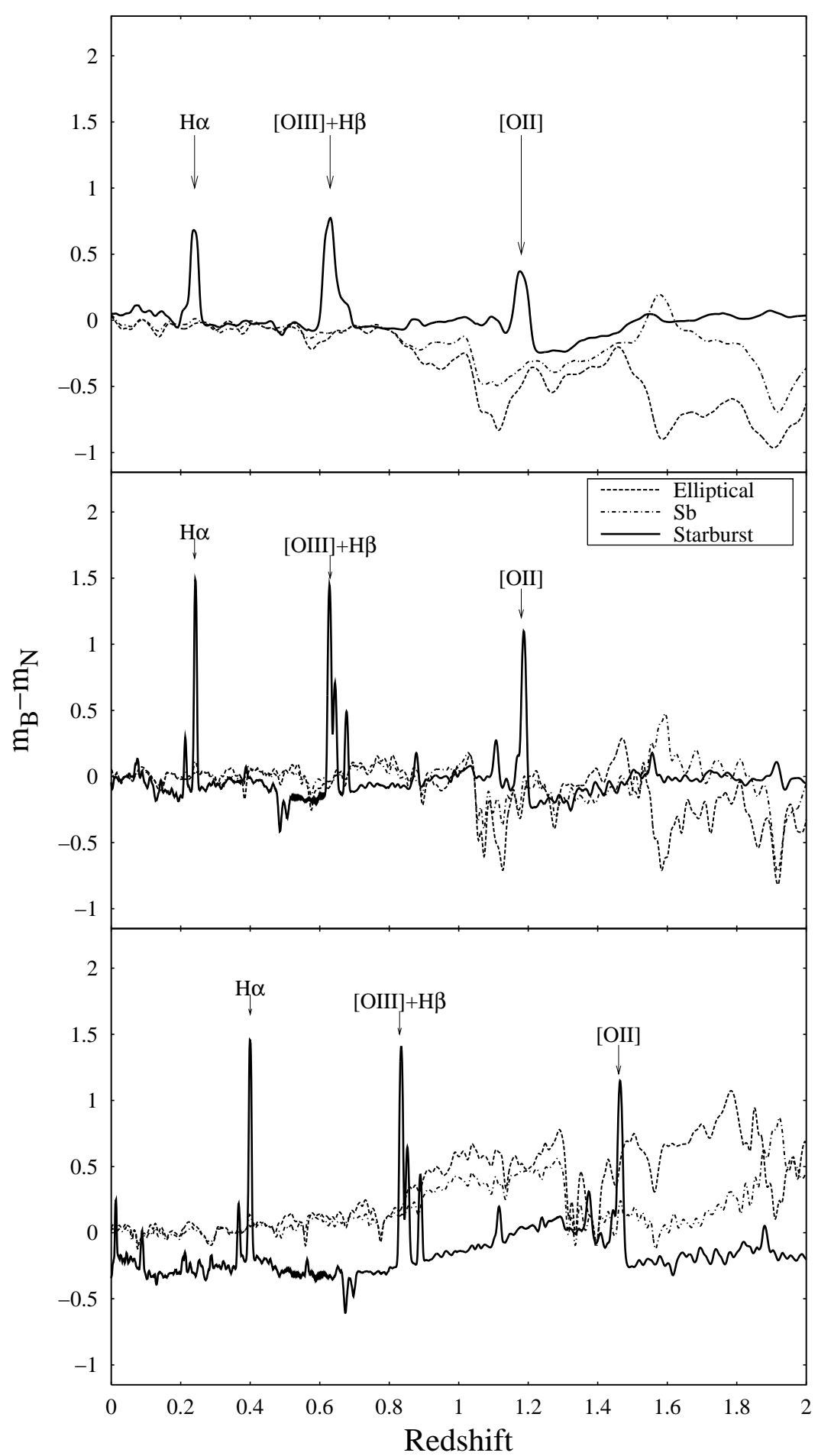

Fig. 7.- Colors of galaxy SEDs for the sample filter sets as a function of redshift. From top to bottom CAFOS8200, WFC8200 and WFC9200. Hubble types templates from Kinney et al. (1996) are plotted. Ellipticals (and bulges) are represented by dashed lines, we use Sb template for early type spirals and S0 (dash-dotted lines) and SB1 starbursts template for starburst and late spirals (Sc) (solid line). The position of the different emission lines is labeled. 


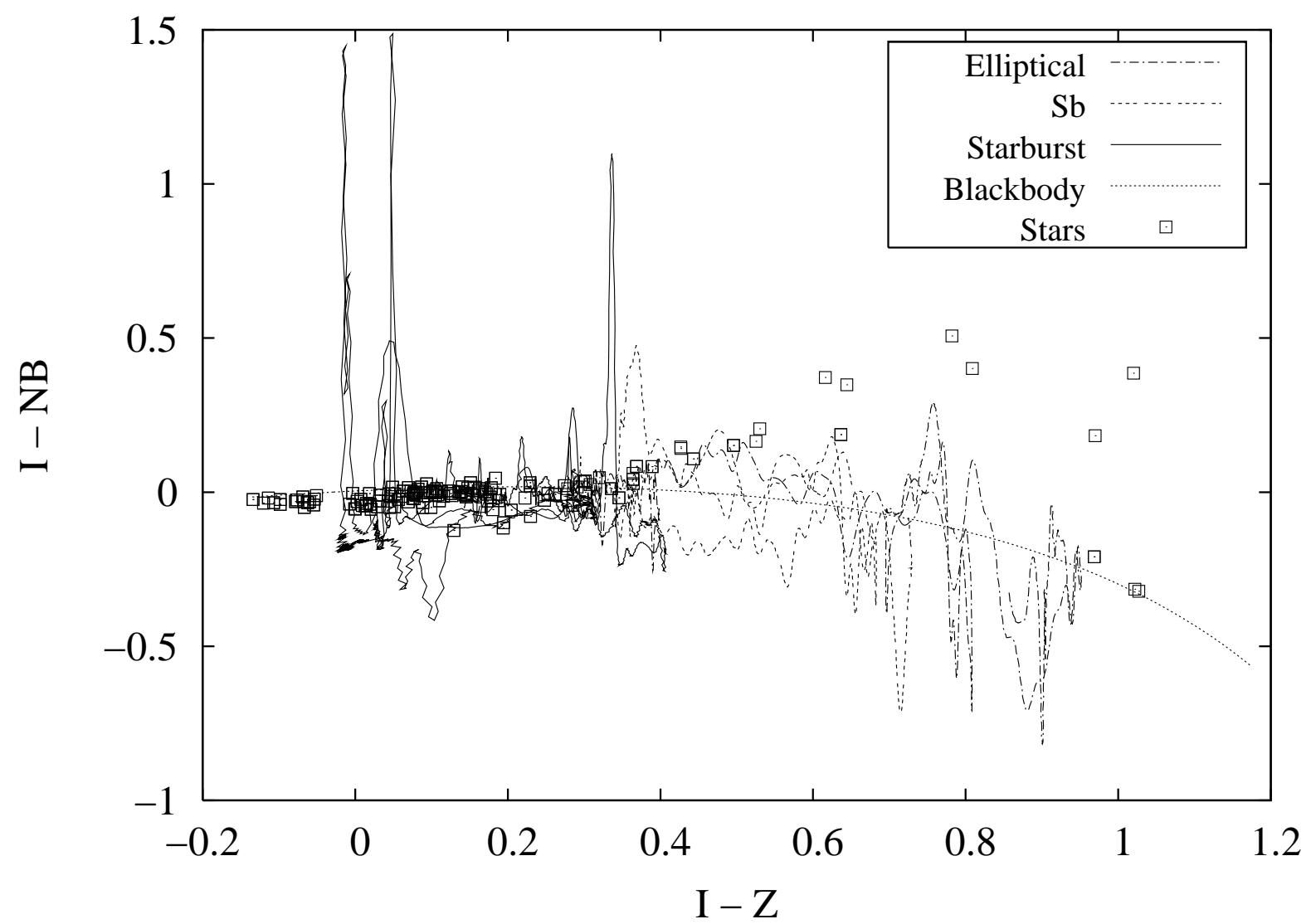

Fig. 8.- $I$ - NB versus $I-Z$ color-color diagram for the WFC8200 filter set. The key for the different types is represented in the box. Starbursts and late type stars exhibit color excess in $I$-NB but have very different colors in $I-Z$. 
with a large range in metal content. The spectral range covers up to $10000 \AA$.

In Fig. 9, the color $m_{\mathrm{B}}-m_{\mathrm{N}}$ as a function of spectral type is plotted. As noted before, stars follow the trend the colors of are dominated by bands of $\mathrm{TiO}$ and $\mathrm{VO}$.

Comparing the colors obtained with CAFOS8200 and WFC8200 we can see that cold stars with molecular bands can be misclassified as ELGs in the case of WFC8200 and WFC9200.

\section{Optimal exposure times}

A critical question that arises when planning observations with narrow-band filters is how long we should expose in the narrow band filter in order to obtain images that can be compared with the corresponding broad band. Exposure simulators available in telescopes usually only cope with broad-band filters.

To be selected in a magnitude color diagram with a signification $n_{\sigma}$, an object with an emission line has to show color excess over the mean distribution of objects at a given magnitude $m_{\mathrm{N}}$. The ratio of fluxes $\mathcal{Q}_{1}$ of an object with emission line has to fulfill:

$$
\mathcal{Q}_{1}>\mu\left[\mathcal{Q}_{\mathrm{O}}\right]+n_{\sigma} \sigma\left[\mathcal{Q}_{\mathrm{O}}\right]
$$

being $\mathcal{Q}_{\mathrm{O}}$ the ratio of fluxes of the objects without emission line and the same narrow-band magnitude that the object with the emission line.

We can approximate $\mu\left[\mathcal{Q}_{\mathrm{O}}\right] \sim \mathcal{Q}_{\mathrm{O}}$ and then:

$$
\frac{1}{n_{\sigma}}\left(\frac{\mathcal{Q}_{1}}{\mathcal{Q}_{\mathrm{O}}}-1\right)>\frac{\sigma\left[\mathcal{Q}_{\mathrm{O}}\right]}{\mathcal{Q}_{\mathrm{O}}}
$$

If we include the SNR via Eq. 44 and Eq. 45 we can obtain the limit condition for the signal-noise ratios:

$$
\rho^{2} \equiv \frac{1}{n_{\sigma}^{2}}\left(\frac{\mathcal{Q}_{1}}{\mathcal{Q}_{\mathrm{O}}}-1\right)^{2}=\mathrm{SNR}_{\mathrm{B}}^{-2}+\mathrm{SNR}_{\mathrm{N}}^{-2}
$$

The SNR depends on the magnitude of the object, the sky background, the aperture where the photometry is measured, some parameters of detector (gain and readout noise) and in the exposure time. In our problem the only free parameters are the exposure times, as the others are fixed by the observing conditions and by the particular emission line object that we wish to detect.

We intend to make the dependence in exposure time explicit. For exposures where the

readout noise of the detector is negligible, the signal-noise ratio goes roughly as the square 


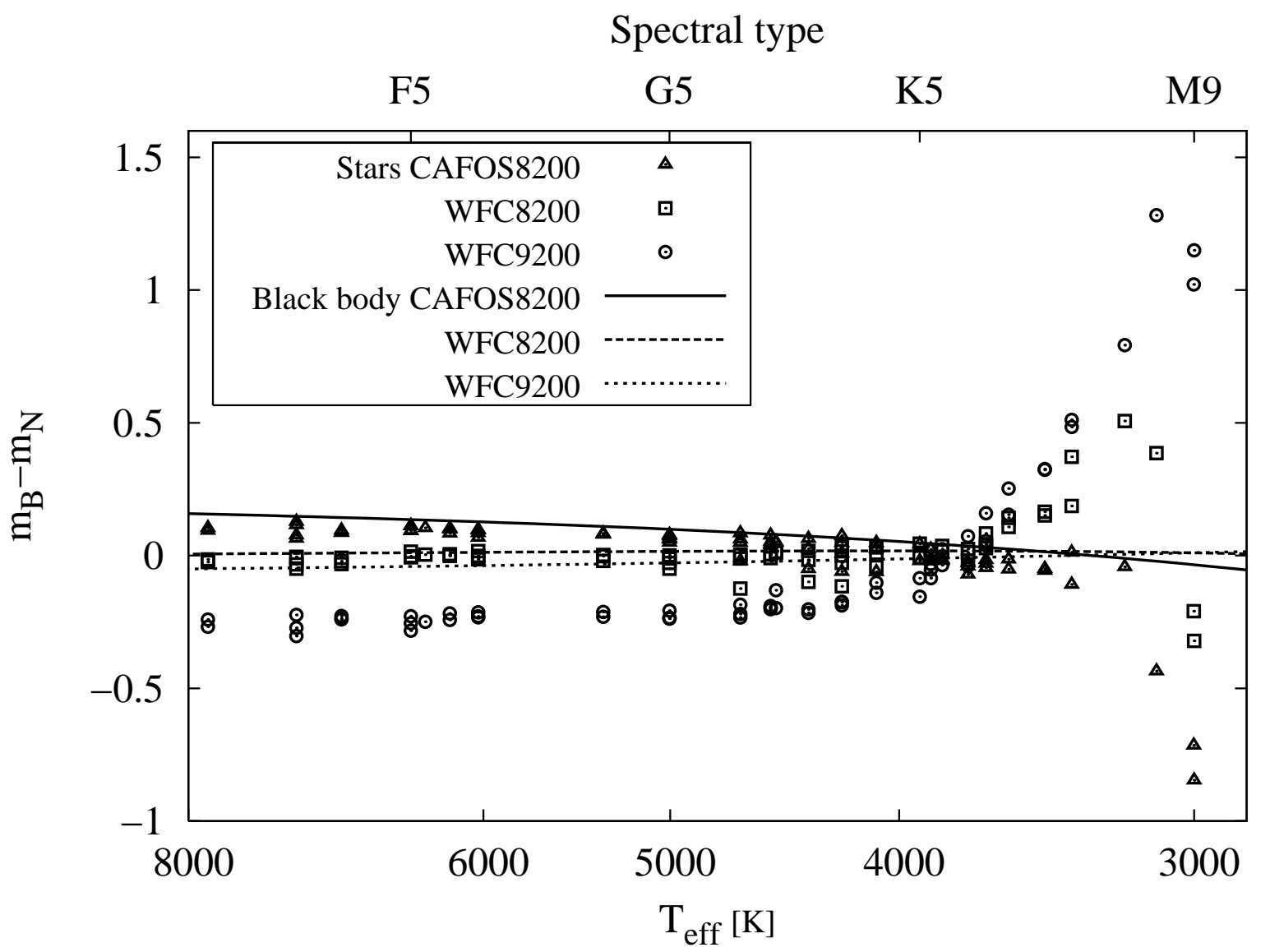

Fig. 9.- Colors of stars of late spectral types in our three sample filters. Stars of late spectral types are also represented with triangles, squares and circles for CAFOS8200, WFC8200 and WFC9200. The colors of a black body on the same filters have been added for comparison and are represented by solid, dashed and dotted lines respectively. The bottom axis represents the effective temperature of the star obtained from the spectral type of the model (top axis). 
root of the exposure time when the other free parameters are fixed. For the broad-band, we comprise in the constant $\alpha$ all the terms not depending on time:

$$
\mathrm{SNR}_{\mathrm{B}}=\alpha \sqrt{t_{\mathrm{B}}}
$$

The signal-noise ratio in both bands is related through the ratio of exposure times. If we start from Eq. 45, we can easily obtain (without any approximation):

$$
\left(\frac{\mathrm{SNR}_{\mathrm{N}}}{\mathrm{SNR}_{\mathrm{B}}}\right)^{2}=b \mathcal{Q}^{2}\left(\frac{t_{\mathrm{N}}}{t_{\mathrm{B}}}\right)\left(\frac{\left(1+q_{\mathrm{B}}\right)+\frac{A G R^{2}}{\dot{N}_{\mathrm{B}} t_{\mathrm{B}}}}{\left(\mathcal{Q}+q_{\mathrm{B}} \mathcal{Q}_{\mathrm{S}}\right)+\frac{A G R^{2}}{b \dot{N}_{\mathrm{B}} t_{\mathrm{N}}}}\right)
$$

For long exposures in both bands, the terms including $R^{2}$ can be neglected and we obtain simply:

$$
\left(\frac{\mathrm{SNR}_{\mathrm{N}}}{\mathrm{SNR}_{\mathrm{B}}}\right)^{2}=b \mathcal{Q}^{2}\left(\frac{t_{\mathrm{N}}}{t_{\mathrm{B}}}\right)\left(\frac{1+q_{\mathrm{B}}}{\mathcal{Q}+q_{\mathrm{B}} \mathcal{Q}_{\mathrm{S}}}\right)=\beta^{2}\left(\frac{t_{\mathrm{N}}}{t_{\mathrm{B}}}\right)
$$

being $\beta$ the following:

$$
\beta^{2}=b \mathcal{Q}^{2}\left(\frac{1+q_{\mathrm{B}}}{\mathcal{Q}+q_{\mathrm{B}} \mathcal{Q}_{\mathrm{S}}}\right)
$$

The parameter $\beta$ has limit cases depending on $q_{\mathrm{B}}$. If $q_{\mathrm{B}} \rightarrow 0$, the noise is dominated by the Poisson noise of the source flux:

$$
\beta^{2}=b \mathcal{Q}
$$

If $q_{\mathrm{B}} \rightarrow \infty$, the equation is dominated by the Poisson noise of the sky background. This is the case for faint objects in the images:

$$
\beta^{2}=b \frac{\mathcal{Q}^{2}}{\mathcal{Q}_{\mathrm{S}}}
$$

We also make explicit the dependence on exposure time of the narrow-band combining Eq. 63 and Eq. 65 to obtain:

$$
\mathrm{SNR}_{\mathrm{N}}=\alpha \beta \sqrt{t_{\mathrm{N}}}
$$

Equation 62 can be written:

$$
\rho^{2}=\frac{1}{\alpha^{2} t_{\mathrm{B}}}+\frac{1}{\alpha^{2} \beta^{2} t_{\mathrm{N}}}
$$

We try to detect 5 - $\sigma$ over the mean objects with $m_{\mathrm{N}}=22$ and $\mathcal{Q}_{1}=1.8$ (this represents a color excess of 0.6 magnitudes ). Assuming a mean color of the objects without emission line of $\mathcal{Q}_{\mathrm{O}}=1$, we immediately compute $\rho=0.16$. 


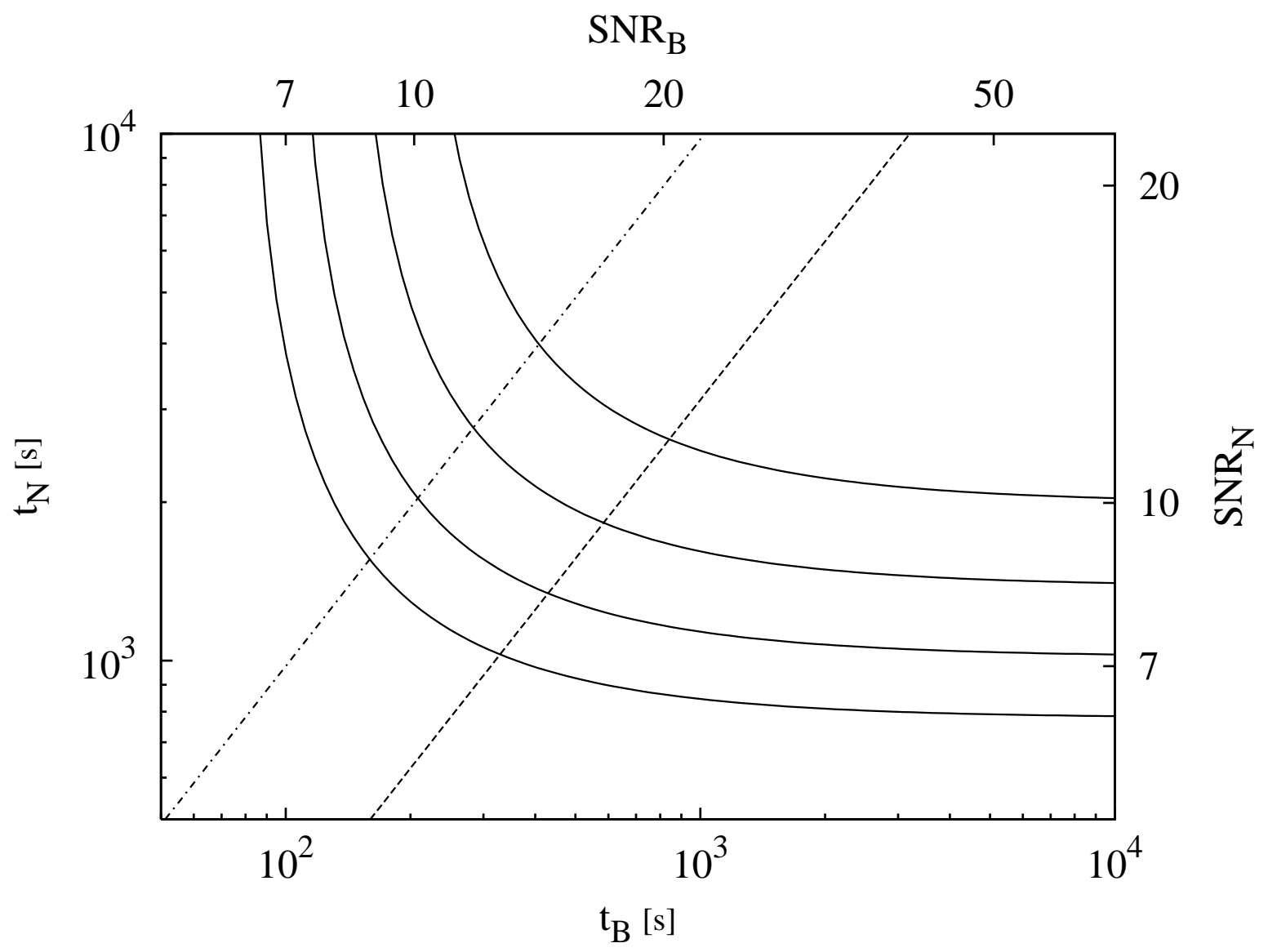

Fig. 10.- Exposure times in broad- $\left(t_{\mathrm{B}}\right)$ and narrow-bands $\left(t_{\mathrm{N}}\right)$ that allow the detection of an object with a given color ratio $\mathcal{Q}_{1}$. The corresponding SNR is also represented. The different curves in solid line correspond to different values of the parameter $\rho$ (see text for the definition). From left to right $\rho=0.16$ (this value is used in examples in text), 0.14 , 0.12 and 0.10 . The dashed line represents the condition of minimum total exposure times and the dash-dotted line the condition of equal SNR in both bands. 
With these filters, the color of the sky background is $\mathcal{Q}_{\mathrm{S}}=0.23$ and $b=0.024$. From the exposure time calculator of the INT WFC, SIGNAL6, we obtain that, for an object of $m_{\mathrm{B}}$ $=22$, with seeing of $1^{\prime \prime}$ and a brightness of the sky background of $19.60 \mathrm{mag} / \mathrm{arcs}^{2}$ (typical for a dark night), the SNR in the broad band is the square root of the exposure time times $\alpha=0.7$.

For the narrow band we compute $\beta$. In this case, $q_{\mathrm{B}}$, computed from the magnitude of the sky background and the magnitude of the objects without emission line, is $\sim 300$. This means that the noise is dominated by the contribution of the sky background and $\beta=0.32$.

In Figure 10 we represent the exposure times in both bands that fulfill the condition in Eq. 70 for different values of $\rho$, from 0.16 (the example we are considering) to 0.10 in steps of 0.02 . For each value there is a set of different exposure times in both bands (given by the curves) that fulfill the conditions.

Each curve is limited by two asymptotic limits, obtained from the condition of infinite SNR in one of the bands:

$$
\begin{aligned}
& \text { For } t_{\mathrm{N}} \rightarrow \infty \quad t_{\mathrm{B}}^{\lim }=\frac{1}{(\rho \alpha)^{2}} \\
& \text { For } t_{\mathrm{B}} \rightarrow \infty \quad t_{\mathrm{N}}^{\lim }=\frac{1}{(\rho \alpha \beta)^{2}}
\end{aligned}
$$

In the current example, the limit exposures are $t_{\mathrm{B}}^{\lim }=80 \mathrm{~s}$ and $t_{\mathrm{N}}^{\lim }=780 \mathrm{~s}$. As the curves lay over their asymptotes, this exposure times are lower limits.

The problem of selecting the optimal exposure times in order to optimize selection of objects with the narrow-band filter still exists at this point. We have to impose one additional condition to extract a pair $t_{\mathrm{B}}, t_{\mathrm{N}}$ from Fig 10. It seems natural to obtain the point that minimizes the total exposure time $t_{\mathrm{B}}+t_{\mathrm{N}}$. This condition is equivalent to minimize the sum of inverse squared SNRs with the constraint of constant $t_{\mathrm{B}}+t_{\mathrm{N}}$.

The exposure times are:

$$
\begin{aligned}
t_{\mathrm{B}} & =\frac{\beta+1}{\rho^{2} \alpha^{2} \beta} \\
t_{\mathrm{N}} & =\frac{\beta+1}{(\rho \alpha \beta)^{2}}=\frac{1}{\beta} t_{\mathrm{B}}
\end{aligned}
$$

\footnotetext{
${ }^{6}$ http://www.ing.iac.es/ds/signal/
} 
In terms of the signal-noise ratio, this last equation can be written as:

$$
\begin{aligned}
\mathrm{SNR}_{\mathrm{B}}^{2} & =\frac{\beta+1}{\beta \rho^{2}} \\
\mathrm{SNR}_{\mathrm{N}}^{2} & =\frac{\beta+1}{\rho^{2}}=\beta \mathrm{SNR}_{\mathrm{B}}^{2}
\end{aligned}
$$

For our particular example, the exposure times are: $t_{\mathrm{B}}=330 \mathrm{~s}$ and $t_{\mathrm{N}}=1030 \mathrm{~s}$. The corresponding signal to noise ratios are $\mathrm{SNR}_{\mathrm{B}}=13$ and $\mathrm{SNR}_{\mathrm{N}}=7$.

From the previous equations we can extract an important conclusion. As typically $\beta<1$, the exposure times in the narrow band are greater than the exposure times in the broad band, but the SNR in the narrow band is lower than the SNR in the broad band. This can be a problem in some extreme cases. The narrow-band image is used normally to detect the objects. Hence, it is necessary to have enough signal-noise ratio in the narrow-band image to be able to detect the objects in it. Note that these exposure times do not account for the overheads proper of observations (dithering, readout time of the detector, etc.).

Other conditions can be applied to Eq. 70 and different exposure times are obtained. If we wish to assure that the SNR is high simultaneously in both images, we can impose the condition of equal SNR to the objects without emission line. Both terms on the right hand side of Eq. [70 are equal. The exposure times are:

$$
\begin{aligned}
t_{\mathrm{B}} & =\frac{2}{\rho^{2} \alpha^{2}} \\
t_{\mathrm{N}} & =\frac{2}{(\rho \alpha \beta)^{2}}=\frac{1}{\beta^{2}} t_{\mathrm{B}}
\end{aligned}
$$

and the common signal-noise ratio is:

$$
\mathrm{SNR}_{\mathrm{B}}^{2}=\mathrm{SNR}_{\mathrm{N}}^{2}=\frac{2}{\rho^{2}}
$$

If we apply the condition of equal SNR to our example, we obtain $t_{\mathrm{B}}=160 \mathrm{~s}$ and $t_{\mathrm{N}}$ $=1560 \mathrm{~s}$, whereas the $\mathrm{SNR}$ in both bands is $\mathrm{SNR}_{\mathrm{B}}=\mathrm{SNR}_{\mathrm{N}}=9$. The total time in this case is $1720 \mathrm{~s}$, that can be compared with the total exposure time of $1360 \mathrm{~s}$ in the optimal case. We have represented in Fig. 10 the lines that correspond to minimum exposure time (dashed) and equal SNR (dash-dotted).

Other possibility is to work in the asymptote of long broad-band exposure times. With the availability of multicolor imaging surveys, deep broad-band public images can be used instead of observing simultaneously in different bands, including the narrow-band. In this 
case, the flux limits are completely determined by the depth of the narrow-band image (in Eq. 62, $\mathrm{SNR}_{\mathrm{B}}^{-2} \ll \mathrm{SNR}_{\mathrm{N}}^{-2}$.

As we have shown, the condition of minimum exposure times provides high enough SNRs to carry out object detection in the narrow-band image. In the SNR obtained is not enough, other conditions can be imposed. The presented exposure times are only lower limits, obtained from the limit condition of Eq. 62. Longer exposure times, if possible, provide better SNRs in both bands that allow determining the line flux and EW of the emission line with lower uncertainties.

The goals of the emission-line survey should be considered when deciding exposure times. Searches for relatively rare objects should survey relatively more area at the cost of getting lower SNR on the narrow-band filters. On the other side, very deep narrow-band survey can select candidates that will have very difficult follow up spectroscopy. In this last case, very deep narrow-band imaging is appropriated, in order to recover line fluxes with very low uncertainty.

\section{Summary and Conclusions}

The main results of this work are as follow:

1. We discuss the contamination of a narrow-band survey, either by galaxies or stars and show different classification schemes that can be used to separate their different contributions. In general, multicolor photometry can be used to classify stars and ELGS at different redshifts. Morphological criteria can also help in the classification.

2. For different narrow-band filters, we compute the mean colors of stars and galaxies. With all the sample filters, the galaxies with emission lines exhibit a color excess and they can be selected as noted by previous authors. Stars of different types can be selected depending on the shapes of the filters involved. A particular analysis is needed in each case. In general, late type stars have molecular bands in the regions studied that can be easily confused with emission lines.

3. The line flux and equivalent width are obtained with different assumptions about the continuum and different filter layouts. line We also confirm that the assumption of an infinitely thin line is appropriate for most physical cases.

4. We study the case of several lines entering simultaneously in the narrow-band filter. This problem arises for $\mathrm{H} \alpha$ plus [NII] $\lambda \lambda 6548,6584$ and [OIII] $\lambda \lambda 4959,5007$ plus $\mathrm{H} \beta$. In 
each case we compute the correction due to the contribution of the other lines. For very narrow filters, using common line ratios can underestimate the line flux, as the additional lines do not enter in the filter.

5. We study the magnitude-color diagram used to select the candidates and we compute an analytic form of the standard deviation of the color of the objects. The standard deviation depends on a number of parameters, namely the exposure times in both bands, the colors of the object and of the background, the surface brightness of the sky and the magnitude of the object. The equation of $\sigma[\mathcal{Q}]$ can help when there are not enough objects detected to calculate the standard deviation from the data available.

6. We show how to compute the redshift range where a candidate can be included in the sample. This range is used to compute the comoving volume associated with each object. Using a global comoving volume for all the object tends to under estimate the volume for faint objects and objects selected near the selection curve.

7. We provide equations to compute the optimal exposure time in the narrow band given the exposure time in the broad band. Given a color ratio for an object, we show the equation that relates the exposure times in both bands needed to effectively select the object.

8. Additionally, if we impose the condition of minimum total exposure time, we obtain directly the exposure times in both bands. These equations are invaluable tools when planing narrow-band observations, as exposure time calculators usually only manage broad-band filters.

This research was supported by the Spanish Programa Nacional de Astronomía y Astrofísica under grant AYA2003-1676.

The authors would like to thank the referee, David Thompson, for insightful comments that improved the paper.

Facilities: CAO:2.2m, ING:Newton .

\section{REFERENCES}

Adelman-McCarthy, J. K., Agüeros, M. A., Allam, S. S., Anderson, K. S. J., Anderson, S. F., Annis, J., Bahcall, N. A., Baldry, I. K., Barentine, J. C., Berlind, A., Bernardi, M., Blanton, M. R., Boroski, W. N., Brewington, H. J., Brinchmann, J., Brinkmann, J., 
Brunner, R. J., Budavári, T., Carey, L. N., Carr, M. A., Castander, F. J., Connolly, A. J., Csabai, I., Czarapata, P. C., Dalcanton, J. J., Doi, M., Dong, F., Eisenstein, D. J., Evans, M. L., Fan, X., Finkbeiner, D. P., Friedman, S. D., Frieman, J. A., Fukugita, M., Gillespie, B., Glazebrook, K., Gray, J., Grebel, E. K., Gunn, J. E., Gurbani, V. K., de Haas, E., Hall, P. B., Harris, F. H., Harvanek, M., Hawley, S. L., Hayes, J., Hendry, J. S., Hennessy, G. S., Hindsley, R. B., Hirata, C. M., Hogan, C. J., Hogg, D. W., Holmgren, D. J., Holtzman, J. A., Ichikawa, S.-i., Ivezić, Ž., Jester, S., Johnston, D. E., Jorgensen, A. M., Jurić, M., Kent, S. M., Kleinman, S. J., Knapp, G. R., Kniazev, A. Y., Kron, R. G., Krzesinski, J., Kuropatkin, N., Lamb, D. Q., Lampeitl, H., Lee, B. C., Leger, R. F., Lin, H., Long, D. C., Loveday, J., Lupton, R. H., Margon, B., Martínez-Delgado, D., Mandelbaum, R., Matsubara, T., McGehee, P. M., McKay, T. A., Meiksin, A., Munn, J. A., Nakajima, R., Nash, T., Neilsen, Jr., E. H., Newberg, H. J., Newman, P. R., Nichol, R. C., Nicinski, T., NietoSantisteban, M., Nitta, A., O’Mullane, W., Okamura, S., Owen, R., Padmanabhan, N., Pauls, G., Peoples, J. J., Pier, J. R., Pope, A. C., Pourbaix, D., Quinn, T. R., Richards, G. T., Richmond, M. W., Rockosi, C. M., Schlegel, D. J., Schneider, D. P., Schroeder, J., Scranton, R., Seljak, U., Sheldon, E., Shimasaku, K., Smith, J. A., Smolčić, V., Snedden, S. A., Stoughton, C., Strauss, M. A., SubbaRao, M., Szalay, A. S., Szapudi, I., Szkody, P., Tegmark, M., Thakar, A. R., Tucker, D. L., Uomoto, A., Vanden Berk, D. E., Vandenberg, J., Vogeley, M. S., Voges, W., Vogt, N. P., Walkowicz, L. M., Weinberg, D. H., West, A. A., White, S. D. M., Xu, Y., Yanny, B., Yocum, D. R., York, D. G., Zehavi, I., Zibetti, S., \& Zucker, D. B. 2006, ApJS, 162,38

Ajiki, M., Mobasher, B., Taniguchi, Y., Shioya, Y., Nagao, T., Murayama, T., \& Sasaki, S. S. 2006a, ApJ, 638, 596

Ajiki, M., Shioya, Y., Taniguchi, Y., Murayama, T., Nagao, T., Sasaki, S. S., Sumiya, R., Morioka, T., Hatakeyama, Y., Yokouchi, A., Takahashi, M. I., \& Koizumi, O. 2006b, PASJ, 58, 113

Ando, M., Ohta, K., Iwata, I., Akiyama, M., Aoki, K., \& Tamura, N. 2006, ApJ, 645, L9

Arnaboldi, M., Freeman, K. C., Okamura, S., Yasuda, N., Gerhard, O., Napolitano, N. R., Pannella, M., Ando, H., Doi, M., Furusawa, H., Hamabe, M., Kimura, M., Kajino, T., Komiyama, Y., Miyazaki, S., Nakata, F., Ouchi, M., Sekiguchi, M., Shimasaku, K., \& Yagi, M. 2003, AJ, 125, 514

Bell, E. F. 2003, ApJ, 586, 794

Bertin, E. \& Arnouts, S. 1996, A\&AS, 117, 393 
Bland-Hawthorn, J. \& Jones, D. H. 1998, Publications of the Astronomical Society of Australia, 15, 44

Bongiovanni, A., Bruzual, G., Magris, G., Gallego, J., García-Dabó, C. E., Coppi, P., \& Sabbey, C. 2005, MNRAS, 359, 930

Bruzual, G. \& Charlot, S. 2003, MNRAS, 344, 1000

Buat, V., Iglesias-Páramo, J., Seibert, M., Burgarella, D., Charlot, S., Martin, D. C., Xu, C. K., Heckman, T. M., Boissier, S., Boselli, A., Barlow, T., Bianchi, L., Byun, Y.I., Donas, J., Forster, K., Friedman, P. G., Jelinski, P., Lee, Y.-W., Madore, B. F., Malina, R., Milliard, B., Morissey, P., Neff, S., Rich, M., Schiminovitch, D., Siegmund, O., Small, T., Szalay, A. S., Welsh, B., \& Wyder, T. K. 2005, ApJ, 619, L51

Calzetti, D. \& Kinney, A. L. 1992, ApJ, 399, L39

Calzetti, D., Kinney, A. L., \& Storchi-Bergmann, T. 1996, ApJ, 458, 132

Cardelli, J. A., Clayton, G. C., \& Mathis, J. S. 1989, ApJ, 345, 245

Castro-Rodríguez, N., Aguerri, J. A. L., Arnaboldi, M., Gerhard, O., Freeman, K. C., Napolitano, N. R., \& Capaccioli, M. 2003, A\&A, 405, 803

Charlot, S. . \& Longhetti, M. 2001, MNRAS, 323, 887

Charlot, S. \& Fall, S. M. 1993, ApJ, 415, 580

Crowther, P. A., Dessart, L., Hillier, D. J., Abbott, J. B., \& Fullerton, A. W. 2002, A\&A, 392,653

Djorgovski, S., Spinrad, H., McCarthy, P., \& Strauss, M. A. 1985, ApJ, 299, L1

Fiorucci, M. \& Munari, U. 2003, A\&A, 401, 781

Fujita, S. S., Ajiki, M., Shioya, Y., Nagao, T., Murayama, T., Taniguchi, Y., Umeda, K., Yamada, S., Yagi, M., Okamura, S., \& Komiyama, Y. 2003, ApJ, 586, L115

Fukugita, M., Shimasaku, K., \& Ichikawa, T. 1995, PASP, 107, 945

Gallagher, J. S., Hunter, D. A., \& Bushouse, H. 1989, AJ, 97, 700

Gallego, J., Zamorano, J., Aragón-Salamanca, A., \& Rego, M. 1995, ApJ, 455, L1

Gallego, J., Zamorano, J., Rego, M., \& Vitores, A. G. 1997, ApJ, 475, 502 
Giavalisco, M., Steidel, C. C., \& Macchetto, F. D. 1996, ApJ, 470, 189

Gil de Paz, A., Boissier, S., Madore, B. F., Seibert, M., Joe, Y. H., Boselli, A., Wyder, T. K., Thilker, D., Bianchi, L., Rey, S. ., Rich, R. M., Barlow, T. A., Conrow, T., Forster, K., Friedman, P. G., Martin, D. C., Morrissey, P., Neff, S. G., Schiminovich, D., Small, T., Donas, J., Heckman, T. M., Lee, Y. ., Milliard, B., Szalay, A. S., \& Yi, S. 2006, ArXiv Astrophysics e-prints astro-ph/0606440

Gil de Paz, A., Madore, B. F., Boissier, S., \& GALEX Science. 2004, American Astronomical Society Meeting Abstracts, 205,

Gronwall, C., Salzer, J. J., Sarajedini, V. L., Jangren, A., Chomiuk, L., Moody, J. W., Frattare, L. M., \& Boroson, T. A. 2004, AJ, 127, 1943

Hartmann, L. W., Huchra, J. P., Geller, M. J., O’Brien, P., \& Wilson, R. 1988, ApJ, 326, 101

Hayes, M. \& Ostlin, G. 2006, ArXiv Astrophysics e-prints

Hippelein, H., Maier, C., Meisenheimer, K., Wolf, C., Fried, J. W., von Kuhlmann, B., Kümmel, M., Phleps, S., \& Röser, H.-J. 2003, A\&A, 402, 65

Hogg, D. W. 1999, ArXiv Astrophysics e-prints astro-ph/99905116

Hopkins, A. M. 2004, ApJ, 615, 209

Hopkins, A. M., Miller, C. J., Nichol, R. C., Connolly, A. J., Bernardi, M., Gómez, P. L., Goto, T., Tremonti, C. A., Brinkmann, J., Ivezić, Ž., \& Lamb, D. Q. 2003, ApJ, 599, 971

Howell, S. B., Koehn, B., Bowell, E., \& Hoffman, M. 1996, AJ, 112, 1302

Hu, E. M., Cowie, L. L., Capak, P., McMahon, R. G., Hayashino, T., \& Komiyama, Y. 2004, AJ, 127,563

Ives, D. J., Tulloch, S., \& Churchill, J. 1996, in Proc. SPIE Vol. 2654, p. 266-276, Solid State Sensor Arrays and CCD Cameras, Constantine N. Anagnostopoulos; Morley M. Blouke; Michael P. Lesser; Eds., 266-276

Iwamuro, F., Motohara, K., Maihara, T., Iwai, J., Tanabe, H., Taguchi, T., Hata, R., Terada, H., Oya, M. G. S., Iye, M., Yoshida, M., Karoji, H., Ogasawara, R., \& Sekiguchi, K. 2000, PASJ, 52, 73 
James, P. A., Shane, N. S., Knapen, J. H., Etherton, J., \& Percival, S. M. 2005, A\&A, 429, 851

Jansen, R. A., Franx, M., \& Fabricant, D. 2001, ApJ, 551, 825

Jones, D. H. \& Bland-Hawthorn, J. 2001, ApJ, 550, 593

Karaali, S., Ak, S. G., Bilir, S., Karataş, Y., \& Gilmore, G. 2003, MNRAS, 343, 1013

Kauffmann, G., Heckman, T. M., Tremonti, C., Brinchmann, J., Charlot, S., White, S. D. M., Ridgway, S. E., Brinkmann, J., Fukugita, M., Hall, P. B., Ivezić, Ž., Richards, G. T., \& Schneider, D. P. 2003, MNRAS, 346, 1055

Kennicutt, R. C. 1983, ApJ, 272, 54

-. 1992, ApJ, 388, 310

-. 1998, ARA\&A, 36, 189

Kewley, L. J. \& Dopita, M. A. 2002, ApJS, 142, 35

Kewley, L. J., Geller, M. J., \& Jansen, R. A. 2004, AJ, 127, 2002

King, D., Rand, R. J., \& Balcells, M. 1994, Spectrum, 2, 20

Kinney, A. L., Calzetti, D., Bohlin, R. C., McQuade, K., Storchi-Bergmann, T., \& Schmitt, H. R. 1996, ApJ, 467, 38

Kodaira, K., Taniguchi, Y., Kashikawa, N., Kaifu, N., Ando, H., Karoji, H., Ajiki, M., Akiyama, M., Aoki, K., Doi, M., Fujita, S. S., Furusawa, H., Hayashino, T., Imanishi, M., Iwamuro, F., Iye, M., Kawabata, K. S., Kobayashi, N., Kodama, T., Komiyama, Y., Kosugi, G., Matsuda, Y., Miyazaki, S., Mizumoto, Y., Motohara, K., Murayama, T., Nagao, T., Nariai, K., Ohta, K., Ohyama, Y., Okamura, S., Ouchi, M., Sasaki, T., Sekiguchi, K., Shimasaku, K., Shioya, Y., Takata, T., Tamura, H., Terada, H., Umemura, M., Usuda, T., Yagi, M., Yamada, T., Yasuda, N., \& Yoshida, M. 2003, PASJ, 55, L17

Lowenthal, J. D., Hogan, C. J., Green, R. F., Caulet, A., Woodgate, B. E., Brown, L., \& Foltz, C. B. 1991, ApJ, 377, L73

Ly, C., Malkan, M. A., Kashikawa, N., Shimasaku, K., Doi, M., Nagao, T., Iye, M., Kodama, T., Morokuma, T., \& Motohara, K. 2006, ArXiv Astrophysics e-prints astro$\mathrm{ph} / 0610846$ 
MacAlpine, G. M., Smith, S. B., \& Lewis, D. W. 1977, ApJS, 34, 95

Macchetto, F., Lipari, S., Giavalisco, M., Turnshek, D. A., \& Sparks, W. B. 1993, ApJ, 404, 511

McCracken, H. J., Radovich, M., Bertin, E., Mellier, Y., Dantel-Fort, M., Le Fèvre, O., Cuillandre, J. C., Gwyn, S., Foucaud, S., \& Zamorani, G. 2003, A\&A, 410, 17

Melbourne, J. \& Salzer, J. J. 2002, AJ, 123, 2302

Mouhcine, M., Bamford, S. P., Aragón-Salamanca, A., Nakamura, O., \& Milvang-Jensen, B. 2006, MNRAS, 369, 891

Mouhcine, M., Lewis, I., Jones, B., Lamareille, F., Maddox, S. J., \& Contini, T. 2005, MNRAS, 362, 1143

Neufeld, D. A. 1990, ApJ, 350, 216

Okamura, S., Yasuda, N., Arnaboldi, M., Freeman, K. C., Ando, H., Doi, M., Furusawa, H., Gerhard, O., Hamabe, M., Kimura, M., Kajino, T., Komiyama, Y., Miyazaki, S., Nakata, F., Napolitano, N. R., Ouchi, M., Pannella, M., Sekiguchi, M., Shimasaku, K., \& Yagi, M. 2002, PASJ, 54, 883

Oke, J. B. 1974, ApJS, 27, 21

Osterbrock, D. E. 1989, Astrophysics of gaseous nebulae and active galactic nuclei (Research supported by the University of California, John Simon Guggenheim Memorial Foundation, University of Minnesota, et al. Mill Valley, CA, University Science Books, 1989, 422 p.)

Pascual, S., Gallego, J., Aragón-Salamanca, A., \& Zamorano, J. 2001, A\&A, 379, 798

Pesch, P. \& Sanduleak, N. 1983, ApJS, 51, 171

Pettini, M. \& Pagel, B. E. J. 2004, MNRAS, 348, L59

Pickles, A. J. 1998, PASP, 110, 863

Rauch, T. 1999, A\&AS, 135, 487

Rhoads, J. E., Xu, C., Dawson, S., Dey, A., Malhotra, S., Wang, J., Jannuzi, B. T., Spinrad, H., \& Stern, D. 2004, ApJ, 611, 59

Rosa-González, D., Terlevich, E., \& Terlevich, R. 2002, MNRAS, 332, 283 
Rudy, R. J., Dimpfl, W. L., Lynch, D. K., Mazuk, S., Venturini, C. C., Wilson, J. C., Puetter, R. C., \& Perry, R. B. 2003, ApJ, 596, 1229

Sabbey, C. N., Oemler, A., Coppi, P., Baltay, C., Bongiovanni, A., Bruzual, G., Garcia, C. E., Musser, J., Rengstorf, A. W., \& Snyder, J. A. 2001, ApJ, 548, 585

Salzer, J. J., MacAlpine, G. M., \& Boroson, T. A. 1989, ApJS, 70, 447

Shimasaku, K., Kashikawa, N., Doi, M., Ly, C., Malkan, M. A., Matsuda, Y., Ouchi, M., Hayashino, T., Iye, M., Motohara, K., Murayama, T., Nagao, T., Ohta, K., Okamura, S., Sasaki, T., Shioya, Y., \& Taniguchi, Y. 2006, PASJ, 58, 313

Smith, M. G. 1975, ApJ, 202, 591

Smith, M. G., Aguirre, C., \& Zemelman, M. 1976, ApJS, 32, 217

Sullivan, M., Treyer, M. A., Ellis, R. S., Bridges, T. J., Milliard, B., \& Donas, J. . 2000, MNRAS, 312, 442

Swinbank, A. M., Smail, I., Chapman, S. C., Blain, A. W., Ivison, R. J., \& Keel, W. C. 2004, ApJ, 617, 64

Taniguchi, Y., Ajiki, M., Nagao, T., Shioya, Y., Murayama, T., Kashikawa, N., Kodaira, K., Kaifu, N., Ando, H., Karoji, H., Akiyama, M., Aoki, K., Doi, M., Fujita, S. S., Furusawa, H., Hayashino, T., Iwamuro, F., Iye, M., Kobayashi, N., Kodama, T., Komiyama, Y., Matsuda, Y., Miyazaki, S., Mizumoto, Y., Morokuma, T., Motohara, K., Nariai, K., Ohta, K., Ohyama, Y., Okamura, S., Ouchi, M., Sasaki, T., Sato, Y., Sekiguchi, K., Shimasaku, K., Tamura, H., Umemura, M., Yamada, T., Yasuda, N., \& Yoshida, M. 2005, PASJ, 57, 165

Thompson, D., Djorgovski, S., \& Trauger, J. 1995, AJ, 110, 963

Tresse, L. \& Maddox, S. J. 1998, ApJ, 495, 691

Tresse, L., Rola, C., Hammer, F., Stasinska, G., Le Fevre, O., Lilly, S. J., \& Crampton, D. 1996, MNRAS, 281, 847

Umeda, K., Yagi, M., Yamada, S. F., Taniguchi, Y., Shioya, Y., Murayama, T., Nagao, T., Ajiki, M., Fujita, S. S., Komiyama, Y., Okamura, S., \& Shimasaku, K. 2004, ApJ, 601,805

van Zee, L., Salzer, J. J., Haynes, M. P., O’Donoghue, A. A., \& Balonek, T. J. 1998, AJ, 116,2805 
Veilleux, S. \& Osterbrock, D. E. 1987, ApJS, 63, 295

Waller, W. H. 1990, PASP, 102, 1217

Wasilewski, A. J. 1983, ApJ, 272, 68

Westra, E., Jones, D. H., Lidman, C. E., Athreya, R. M., Meisenheimer, K., Wolf, C., Szeifert, T., Pompei, E., \& Vanzi, L. 2005, A\&A, 430, L21

Westra, E., Jones, D. H., Lidman, C. E., Meisenheimer, K., Athreya, R. M., Wolf, C., Szeifert, T., Pompei, E., \& Vanzi, L. 2006, A\&A, 455, 61

Wolf, C., Meisenheimer, K., Röser, H.-J., Beckwith, S. V. W., Chaffee, Jr., F. H., Fried, J., Hippelein, H., Huang, J.-S., Kümmel, M., von Kuhlmann, B., Maier, C., Phleps, S., Rix, H.-W., Thommes, E., \& Thompson, D. 2001, A\&A, 365, 681

Yan, L., McCarthy, P. J., Freudling, W., Teplitz, H. I., Malumuth, E. M., Weymann, R. J., \& Malkan, M. A. 1999, ApJ, 519, L47

Zamorano, J., Gallego, J., Rego, M., Vitores, A. G., \& Alonso, O. 1996, ApJS, 105, 343

Zamorano, J., Rego, M., Gallego, J. G., Vitores, A. G., González-Riestra, R., \& RodríguezCaderot, G. 1994, ApJS, 95, 387 